\title{
Impact of Cell Size Effect on Nutrient-Phytoplankton Dynamics
}

\author{
Tiancai Liao, ${ }^{1,2}$ Hengguo Yu $\mathbb{D}^{2,3}$ Chuanjun Dai, ${ }^{2,4}$ and Min Zhao $\mathbb{D}^{2,4}$ \\ ${ }^{1}$ Key Laboratory of Saline-Alkali Vegetation Ecology Restoration in Oil Field, Ministry of Education, \\ Northeast Forestry University, Harbin, Heilongjiang 150040, China \\ ${ }^{2}$ Zhejiang Provincial Key Laboratory for Water Environment and Marine Biological Resources Protection, Wenzhou University, \\ Wenzhou, Zhejiang 325035, China \\ ${ }^{3}$ Department of Mathematics and Information Science, Wenzhou University, Wenzhou, Zhejiang 325035, China \\ ${ }^{4}$ College of Life and Environmental Science, Wenzhou University, Wenzhou, Zhejiang 325035, China
}

Correspondence should be addressed to Min Zhao; zmcn@tom.com

Received 17 September 2019; Accepted 9 November 2019; Published 28 November 2019

Guest Editor: Tomas Veloz

Copyright (C) 2019 Tiancai Liao et al. This is an open access article distributed under the Creative Commons Attribution License, which permits unrestricted use, distribution, and reproduction in any medium, provided the original work is properly cited.

In this paper, a nutrient-phytoplankton model, which is described by a system of ordinary differential equations incorporating the effect of cell size, and its corresponding stochastic differential equation version are studied analytically and numerically. A key advantage of considering cell size effect is that it can more accurately reveal the intrinsic law of interaction between nutrient and phytoplankton. The main purpose of this paper is to research how cell size affects the nutrient-phytoplankton dynamics within the deterministic and stochastic environments. Mathematically, we show that the existence and stability of the equilibria in the deterministic model can be determined by cell size: the smaller or larger cell size can lead to the disappearance of the positive equilibrium, but the boundary equilibrium always exists and is globally asymptotically stable; the intermediate cell size is capable to drive the positive equilibrium to appear and be globally asymptotically stable, whereas the boundary equilibrium becomes unstable. In the case of the stochastic model, the stochastic dynamics including the stochastic extinction, persistence in the mean, and the existence of ergodic stationary distribution is found to be largely dependent on cell size and noise intensity. Ecologically, via numerical simulations, it is found that the smaller cell size or larger cell size can result in the extinction of phytoplankton, which is similar to the effect of larger random environmental fluctuations on the phytoplankton. More interestingly, it is discovered that the intermediate cell size is the optimal size for promoting the growth of phytoplankton, but increasing appropriately the cell size can rapidly reduce phytoplankton density and nutrient concentrations at the same time, which provides a possible strategy for biological control of algal blooms.

\section{Introduction}

Phytoplankton blooms, which can negatively affect the aquatic ecosystems, human health, marine fisheries, and local economy, are growing in frequency, magnitude, and duration globally in recent years [1, 2]. For example, in 2007, serious harmful algal blooms broke out in Lake Taihu, resulting in about 2 million people in Wuxi city short of drinking water for more than a week [3]. In 2011, Lake Erie experienced a record-breaking harmful algal bloom, with a peak intensity more than three times the previously observed algal blooms [4]. These have stimulated a number of scholars to study the dynamics of phytoplankton growth by many different ways, in order to explore the possible mechanisms underlying the occurrence or termination of these blooms. Nevertheless, such a mechanism in response to the phenomena of blooms is still under investigation due to the diversity and complexity of influencing factors that can affect the growth of phytoplankton in the real aquatic ecological environments. Hence, the research in seeking for some key factors affecting the growth mechanisms of phytoplankton is currently of great interest.

However, in the real aquatic environments, the growth of phytoplankton is generally influenced by many biotic and abiotic factors, such as light [5], cell size [6], climate [7], grazer [8], carbon dioxide [9], nutrient [10], and 
temperature [11], which make it difficult to determine a clear mechanism of phytoplankton blooms only through experimental studies. Actually, many ecologists, biologists, and biomathematicians increasingly realize that a mathematical model is a powerful tool for exploring biological and physical processes on the dynamic mechanisms of phytoplankton growth in relation to different factors qualitatively and quantitatively $[12,13]$, as the research results can help us to find out the key factors that may induce the blooms of phytoplankton but are difficult to predict in the experimental analysis, to answer that what the growth mechanism of phytoplankton is, to predict possibly when the phytoplankton blooms will occur, and to determine the optimal strategy for possible control of phytoplankton blooms [14-25]. The application of mathematical models in other research fields, such as investigating other predator-prey dynamics or infectious disease dynamics, can be found in [26-37].

In 1949, Riley et al. [38] first used the mathematical model to study the nutrient-plankton dynamics, which leads to the formulation of a growing number of mathematical models to describe the nutrient-phytoplankton dynamics or nutrient-plankton dynamics, and many dynamic mechanisms of phytoplankton growth response to various factors have been revealed [14, 15, 22, 39-47]. For example, Chen et al. [43] showed that the proper control of the ratio for nitrogen and phosphorus can more effectively control and eliminateblue-green algae blooms. $\mathrm{Pal}$ et al. [44] indicated that the toxin produced by phytoplankton plays a crucial role in the termination of planktonic blooms when the nutrient concentration is very high. Dai et al. [39] signified that time delay not only induces instability of a positive equilibrium but also promotes the formation of patchiness. Chatterjee et al. [41] observed that the nutrient input rate specially caused by artificial eutrophication has a great influence on controlling the planktonic blooms and maintaining stability around the coexistence equilibrium. These excellent results have made a great contribution to the research processes related to the possible mechanisms for the formation or termination of phytoplankton blooms, which in turn indicate that the modeling studies are an alternative, effective, and feasible method to investigate some important factors that may reveal the nature of the blooms of phytoplankton occurring frequently in various water bodies around the world.

Most of the existing mathematical modeling studies on the nutrient-phytoplankton dynamics or nutrient-plankton dynamics usually assume that the nutrient uptake rate, phytoplankton sinking rate, phytoplankton growth rate, and so on are independent of cell size $[12,14,15,22$, 39-45], which have, in part, been considered to be unrealistic because the factor of cell size is capable to significantly affect the dynamic mechanisms of phytoplankton growth [48]. In fact, cell size is a master functional trait that virtually affects every aspect of phytoplankton biology at the cellular, population, and community levels [49]. Hence, the cell size of phytoplankton not only defines their metabolic activity, growth rates, and numerical abundance but also strongly affects their contributions to biogeochemical cycles via size-dependent sinking and influences community structure and dynamics via sizedependent species interactions [50-53]. A recent remarkable experimental work, in this research direction, was carried out by Marañón et al. [6], where they further determined experimentally that the growth rate, metabolic rate, and nutrient uptake rate of phytoplankton are sizedependent by using cultures of 22 species of marine phytoplankton from five phyla, ranging from 0.1 to $10^{6} \mu \mathrm{m}^{3}$ in cell volume. Furthermore, by performing an in situ test of Raven's prediction that there is a reversal of the relationship between cell size and maximum achievable growth rate in unicellular algae at the low end of size classes, Bec et al. [54] found that the biomass specific production and growth rates are similar in both small and large cells but peak at intermediate cell sizes. Nevertheless, despite these facts, there is little theoretical explanation on how cell size affects the growth mechanism of phytoplankton. In recent years, a review of relevant literature shows that several plankton models taking into account the effect of cell size have been reported [55-57]. More specifically, $\mathrm{Pu}$ et al. [56] indicated that the coevolutionary dynamics of plankton is closely related to cell size. Zhao et al. [57] found that the cell size can significantly affect the growth and reproduction of phytoplankton. The cell size plays an important role in the dynamics of interacting phytoplankton, which has been clearly demonstrated by these recent studies.

In spite of the importance of cell size, a comprehensive understanding towards the dynamic mechanism for phytoplankton growth in relation to cell size is still lacking. Hence, naturally, some questions arise. How cell size affects the nutrient concentration and phytoplankton density? Can the cell size affect the existence and stability of the equilibria? In order to find out the answers to these questions, motivated by these works $[12,55,56,58]$, in the present study, we propose and investigate a nutrientphytoplankton model, incorporating the effect of cell size, as follows:

$$
\left\{\begin{array}{l}
\frac{\mathrm{d} N}{\mathrm{~d} t}=I-\frac{\mu(x) Q(x) N P}{a+N}-m N, \\
\frac{\mathrm{d} P}{\mathrm{~d} t}=\frac{\mu(x) N P}{a+N}-s(x) P-\mathrm{d} P,
\end{array}\right.
$$

subject to the initial conditions $N(0)=N_{0} \geq 0$ and $P(0)=P_{0} \geq 0$, where $N(t)$ and $P(t)$ are the concentration of nutrient and the density of phytoplankton at time $t$, respectively. Here, we assume that $I$ is the input rate of nutrient from the environments, $m$ is the removal rate of nutrient, and $d$ is maximum mortality rate of phytoplankton. Let $x$ be the cell size of phytoplankton; we suppose that $\mu(x)=\left(x /\left(a_{1} x^{2}+a_{2} x+a_{3}\right)\right)[55]$ is the maximum specific growth rate of phytoplankton as a function of cell size, and $Q(x)=\beta x^{3}$ [55] signifies the nutrient quota which is proportional to the cube of cell size, where $a_{i}(i=1,2,3)$ are positive empirical constants and $\beta$ denotes the phytoplankton nutrient quota coefficient. The nutrient uptake rate 
of phytoplankton is assumed to depend on $\mu(x)$ and $Q(x)$. That is, the term $\mu(x) Q(x) N P /(a+N)$ is taken as a Michaelis-Menten function that may provide a more realistic reflection of nutrient uptake dynamics [12], leading to a decrease in nutrient concentration, where $a$ is the halfsaturation constant of nutrient. Accordingly, the term $\mu(x) N P /(a+N)$ is the conversion efficiency of nutrient uptake by phytoplankton, which means that phytoplankton absorbs nutrient to reproduce offspring and increase the number of phytoplankton. Suppose that the sinking rate of phytoplankton is proportional to the square of cell size, i.e., $s(x)=\alpha x^{2}$ [55], where $\alpha$ is a constant whose value is affected by the density of the water and the algal cell as well as the viscosity of the water. All the parameters mentioned above are assumed to be positive. From a biological viewpoint, we need to ensure that $(\mathrm{d} P / \mathrm{d} t)>0$, so it is assumed that $\mu(x)-$ $s(x)-d>0$ be always established by default in the whole paper.

On the other hand, in the natural world, the aquatic environment in which phytoplankton lives is always uncertain and random because of the environmental noise disturbances [59], such as unpredictable radiation, light availability, and water temperature variation. Hence, the parameters contained in deterministic models are usually assumed to be invariant constants, which have obvious limitations in modeling the natural ecological systems. Actually, most of the natural phenomena do not strictly follow deterministic laws, but rather oscillate randomly about some average behaviors. A fact further pointed out by May [60] is that the birth rate, carrying capacity, and other parameters involved the model should exhibit random fluctuation to a greater or lesser extent because of the environmental noise effects. Consequently, using stochastic differential equation models may reveal the dynamic mechanisms of phytoplankton growth more accurately compared to their deterministic counterparts. For these reasons, some authors recently introduced environmental noise fluctuations into the aquatic plankton models to study noise influences on the interplay and growth of phytoplankton [61-64]. For example, Yu et al. [61] indicated that environmental fluctuations play a key role in the termination of algal blooms. Camara et al. [63] suggested that stochastic environmental constraints have positive and negative effects on the life of Daphnia and algae populations. Obviously, the environmental fluctuations have a vital role in the dynamics of phytoplankton growth. In other words, stochastic environmental disturbance effects should be considered when mathematical models are used to study and model the ecological systems in nature.

Consequently, we introduce white noise perturbations into the deterministic model (1). In the existing literature, there are many different ways to incorporate the noise fluctuations into the ecological systems. In this study, by following the method in [65], we assume that stochastic environmental fluctuations mainly affect the growth of phytoplankton $\mu(x)$. In this way, $\mu(x)$ changes to a random variable $\overline{\mu(x)}$, and $\overline{\mu(x)}=\mu(x)+\delta \dot{B}(t)$, where $B(t)$ is a standard Brownian motion defined on a complete probability space $\left(\Omega, \mathscr{F},\left(\mathscr{F}_{t}\right)_{t \geq 0}, \mathbb{P}\right), \dot{B}(t)$ indicates the white noise, and $\delta$ represents the intensity of the white noise. Therefore, by replacing $\mu(x)$ in the deterministic model (1) with $\mu(x)+\delta \dot{B}(t)$, i.e.,

$$
\begin{gathered}
-\frac{m u(x) Q(x) N P}{a+N} \longrightarrow-\frac{\mu(x) Q(x) N P}{a+N}-\frac{\delta \mu(x) Q(x) N P}{a+N} \dot{B}(t), \\
\frac{\mu(x) N P}{a+N} \longrightarrow \frac{\mu(x) N P}{a+N}+\frac{\delta \mu(x) N P}{a+N} \dot{B}(t),
\end{gathered}
$$

and then, model (1) becomes

$$
\left\{\begin{array}{l}
\mathrm{d} N=\left(I-\frac{\mu(x) Q(x) N P}{a+N}-m N\right) \mathrm{d} t-\delta \frac{\mu(x) Q(x) N P}{a+N} \mathrm{~d} B(t), \\
\mathrm{d} P=\left(\frac{\mu(x) N P}{a+N}-s(x) P-\mathrm{d} P\right) \mathrm{d} t+\delta \frac{\mu(x) N P}{a+N} \mathrm{~d} B(t) .
\end{array}\right.
$$

In the plankton ecology, the persistence and extinction of phytoplankton, which determine whether the phytoplankton is survival or not in the future, are two important topics. However, in the existing literature, the issues on how cell size affects the stochastic extinction and persistence of phytoplankton and how cell size affects the existence of ergodic stationary distribution of phytoplankton that denotes the weak stability of phytoplankton in a stochastic sense, and so on remain largely unanswered. In this paper, we did some works in this research area by studying the stochastic dynamics of model (3).

The main purpose of this paper is to investigate how cell size affects the nutrient-phytoplankton dynamics within the deterministic and stochastic environments by trying to answer the questions proposed in this paper.

In order to facilitate the mathematical analysis for model (1) and model (3) in the following paper, we define

$$
X(x)=\frac{\mu(x)-s(x)-d}{d+s(x)}>0,
$$

and we write $X(x)$ as $X$ for simplicity and convenience. The rest of this article is organized as follows: in Section 2, we explore the dynamics of model (1). Section 3 is devoted to studying the dynamics of model (3). In Section 4, we carry out the numerical simulations to verify the analytical results. Section 5 focuses on giving a brief discussion and the summary of the main results.

\section{Dynamics of the Deterministic Model (1)}

In this section, we mainly focus on investigating the positivity and boundedness of the solutions, as well as studying the existence and stability of the possible equilibria in model (1).

2.1. Positivity and Boundedness of the Solutions. Now, we first present the positivity of the solutions.

Lemma 1. For any initial value $(N(0), P(0))=\left(N_{0}, P_{0}\right)>0$, all the solutions of model (1) are positive invariant. 
Proof. In model (1), we have $N(t)>0$ and $P(t)>0$ for any $t \in[0, B]$, where $B$ is any positive real number. Suppose this is not true, then there exists $B_{\tau} \in(0, B)$ such that, for any $t \in\left[0, B_{\tau}\right], N(t)>0, P(t)>0$, and either $N\left(B_{\tau}\right)=0$ or $P\left(B_{\tau}\right)=0$. Based on the nutrient and phytoplankton equations in model (1), we can obtain

$$
\begin{aligned}
& N(t)=N(0) \exp \left[\int_{0}^{t}\left(\frac{I}{N(s)}-\frac{\mu(x) Q(x) P(s)}{a+N(s)}-m\right) \mathrm{d} s\right] \\
& P(t)=P(0) \exp \left[\int_{0}^{t}\left(\frac{\mu(x) N(s)}{a+N(s)}-d-s(x)\right) \mathrm{d} s\right] .
\end{aligned}
$$

Since $(N(t), P(t))$ are defined and continuous in $\left[0, B_{\tau}\right]$, there exists $\varrho \geq 0$ such that for all $t \in\left[0, B_{\tau}\right]$,

$$
\begin{aligned}
N(t) & =N(0) \exp \left[\int_{0}^{t}\left(\frac{I}{N(s)}-\frac{\mu(x) Q(x) P(s)}{a+N(s)}-m\right) \mathrm{d} s\right] \\
& \geq N(0) \exp \left(-B_{\tau} \varrho\right), \\
P(t) & =P(0) \exp \left[\int_{0}^{t}\left(\frac{\mu(x) N(s)}{a+N(s)}-d-s(x)\right) \mathrm{d} s\right] \\
& \geq P(0) \exp \left(-B_{\tau} \varrho\right) .
\end{aligned}
$$

Obviously, if $(N(0), P(0))=\left(N_{0}, P_{0}\right)>0$ and let $t \longrightarrow B_{\tau}$, we have $N\left(B_{\tau}\right) \geq N(0) \exp \left(-B_{\tau} \varrho\right)>0$ and $P\left(B_{\tau}\right) \geq P(0) \exp \left(-B_{\tau} \varrho\right)>0$, which contradict the fact that either $N\left(B_{\tau}\right)=0$ or $P\left(B_{\tau}\right)=0$. Hence, for all $t \in\left[0, B_{\tau}\right]$, $N(t)>0$ and $P(t)>0$. This completes the proof.

Next, we study the boundedness of the solutions. Let $W(t)=N(t)+Q(x) P(t)$, then we have

$$
\begin{aligned}
\frac{\mathrm{d} W}{\mathrm{~d} t}= & \frac{\mathrm{d} N}{\mathrm{~d} t}+\frac{\mathrm{d} P}{\mathrm{~d} t} \\
= & I-\frac{\mu(x) Q(x) N P}{a+N}-m N+\frac{\mu(x) Q(x) N P}{a+N}-d Q(x) P \\
& -Q(x) s(x) P \\
= & I-m N-(d+s(x)) Q(x) P \\
\leq & I-e W,
\end{aligned}
$$

where $e=\min \{m,(d+s(x))\}$. Thus, we have $(\mathrm{d} W / \mathrm{d} t)+$ $e W \leq I$. By using the theory of differential inequality [66], for all $t \geq T \geq 0$, we have

$$
0 \leq W(t) \leq \frac{I}{e}-\left(\frac{I}{e}-W(T)\right) \exp (-e(t-T)) .
$$

Hence,

$$
\lim _{t \longrightarrow \infty} \sup (N(t)+Q(x) P(t)) \leq \frac{I}{e} .
$$

So, we can obtain the theorem as follows.
Theorem 1. All the solutions of model (1) that start in $R_{+}^{2}$ are uniformly bounded.

2.2. Existence and Stability of Equilibria. This section is devoted to investigating the existence and stability of the possible equilibria in model (1). Now, we first consider the existence of possible equilibria in model (1).

From the nutrient and phytoplankton equations in model (1), by performing a simple computation, we have that model (1) possesses two equilibria as follows:

(a) The boundary equilibrium $E_{1}=(I / m, 0)$ always exists

(b) The positive equilibrium $E_{*}=(a / X,(I X-m a) /$ $(Q(x)(\mu(x)-s(x)-d)))$, provided $X>(m a / I)$

Next, we deal with the stability of the equilibria in model (1). Through direct calculations, the Jacobian matrix of model (1) at equilibrium $E(N, P)$ is

$$
J_{E}=\left[\begin{array}{cc}
-\frac{\mu(x) Q(x) a P}{(a+N)^{2}}-m & -\frac{\mu(x) Q(x) N}{a+N} \\
\frac{\mu(x) a P}{(a+N)^{2}} & \frac{N \mu(x)-(d+s(x))(a+N)}{a+N}
\end{array}\right] .
$$

Obviously, the variational matrix of model (1) at the boundary equilibrium $E_{1}$ is

$$
J_{E_{1}}=\left[\begin{array}{cc}
-m & -\frac{I \mu(x) Q(x)}{I+m a} \\
0 & \frac{(I X-m a)(d+s(x))}{m a+I}
\end{array}\right],
$$

and the two eigenvalues of $J_{E_{1}}$ are $\lambda_{1}=-m<0$ and $\lambda_{2}=(I X-m a)(d+s(x)) /(m a+I)$. Hence, the stability of $E_{1}$ depends on the sign of $\lambda_{2}$. That is, if $X<(m a / I), E_{1}$ is locally asymptotically stable and is unstable if $X>(\mathrm{ma} / \mathrm{I})$.

The variational matrix of model (1) at the positive equilibrium $E_{*}$ is given by

$$
J_{E_{*}}=\left[\begin{array}{ll}
J_{11} & J_{12} \\
J_{21} & J_{22}
\end{array}\right]=\left[\begin{array}{cc}
-\frac{X(X I-m a)}{a(X+1)}-m & -\frac{\mu(x) Q(x)}{X+1} \\
\frac{X(X I-m a)}{a(X+1) Q(x)} & 0
\end{array}\right],
$$

where $J_{11}<0, J_{12}<0$, and $J_{21}>0$. Then, it is not difficult to derive the characteristic equation of the positive equilibrium $E_{*}$ which is $\lambda^{2}-\operatorname{tr}\left(J_{E_{*}}\right) \lambda+\operatorname{det}\left(J_{E_{*}}\right)=0$, where $\operatorname{tr}\left(J_{E_{*}}\right)=$ $J_{11}<0$ and $\operatorname{det}\left(J_{E_{*}}\right)=-J_{21} J_{12}>0$. Based on the RouthHurwitz criterion, the positive equilibrium $E_{*}$ in model (1) is locally asymptotically stable when it exists.

Hence, we have the following result. 
Theorem 2. For model (1), if $X<(\mathrm{ma} / \mathrm{I})$, there exists the only boundary equilibrium $E_{1}$, which is locally asymptotically stable; if $X>(\mathrm{ma} / \mathrm{I})$, the positive equilibrium $E_{*}$ appears and is locally asymptotically stable, but the boundary equilibrium $E_{1}$ becomes unstable.

In what follows, we provide some results concerning the global stability of the equilibria.

Theorem 3. If $X<(\mathrm{ma} / \mathrm{I})$ holds, the boundary equilibrium of model (1) is globally asymptotically stable.

Proof. Letting $E_{1}=(I / M, 0)=\left(N_{1}, P_{1}\right)$ and defining $f(y)=\mu(x) Q(x) y /(a+y)$ with $y \in R$, we consider the following function:

$$
V_{1}(N, P)=\int_{N_{1}}^{N} \frac{f(s)-f(N)}{f(s)} \mathrm{d} s+k P,
$$

where $k$ is a positive constant that will be determined in the paper later.

The derivative of $V_{1}$ with respect to the time $t$ along the solutions of model (1) is

$$
\begin{aligned}
\frac{\mathrm{d} V_{1}}{\mathrm{~d} t}= & \frac{f(N)-f\left(N_{1}\right)}{f(N)} \frac{\mathrm{d} N}{\mathrm{~d} t}+k \frac{\mathrm{d} P}{\mathrm{~d} t} \\
= & \frac{f(N)-f\left(N_{1}\right)}{f(N)}\left(-m\left(N-N_{1}\right)-f(N) P\right) \\
& +\frac{k}{Q(x)} f(N) P-(s(x)+d) k P \\
= & \frac{-m\left(f(N)-f\left(N_{1}\right)\right)\left(N-N_{1}\right)}{f(N)}+\left(\frac{k}{Q(x)}-1\right) f(N) P \\
& +\left(f\left(N_{1}\right)-(d+s(x)) k\right) P .
\end{aligned}
$$

Now, we obtain $k=Q(x)>0$, and then, the above equation becomes

$$
\begin{aligned}
\frac{\mathrm{d} V_{1}}{\mathrm{~d} t}= & \frac{-m\left(f(N)-f\left(N_{1}\right)\right)\left(N-N_{1}\right)}{f(N)} \\
& +\left(f\left(N_{1}\right)-(d+s(x)) Q(x)\right) P .
\end{aligned}
$$

Notice that $f(y)>0$ is an increasing function for any $y>0$, and in view of Lemma 1, if $f\left(N_{1}\right)-(d+s(x)) Q(x)<0$, that is, $X<(m a / I)$, one can get $\left(\mathrm{d} V_{1} / \mathrm{d} t\right) \leq 0$ and $\left(\mathrm{d} V_{1} / \mathrm{d} t\right)=0$ if and only if $(N, P)=\left(N_{1}, P_{1}\right)$. Hence, Lyapunov-LaSalle's invariance principle implies the global asymptotic stability of $E_{1}$. This completes the proof.

Theorem 4. If $X>(m a / I)$, the positive equilibrium $E_{*}$ in model (1) is globally asymptotically stable.

Proof. We consider such a function:

$$
V_{2}(N, P)=\int_{N_{*}}^{N} \frac{f(s)-f\left(N_{*}\right)}{f(s)} \mathrm{d} s+Q(x) \int_{P_{*}}^{P} \frac{s-P_{*}}{s} \mathrm{~d} s,
$$

where the function $f(y)(y \in R)$ is the same as the definition above.

The derivative of $V_{2}$ with respect to the time $t$ along the solutions of model (1) is

$$
\begin{aligned}
\frac{\mathrm{d} V_{2}}{\mathrm{~d} t}= & \frac{f(N)-f\left(N_{1}\right)}{f(N)} \frac{\mathrm{d} N}{\mathrm{~d} t}+Q(x) \frac{P-P_{*}}{P} \frac{\mathrm{d} P}{\mathrm{~d} t} \\
= & \frac{f(N)-f\left(N_{*}\right)}{f(N)}\left[-P_{*}\left(f(N)-f\left(N_{*}\right)\right)-f(N)\left(P-P_{*}\right)\right. \\
& \left.-m\left(N-N_{*}\right)\right]+\left(P-P_{*}\right)\left(f(N)-f\left(N_{*}\right)\right) \\
= & -\frac{P_{*}\left(f(N)-f\left(N_{*}\right)\right)^{2}}{f(N)}-\frac{m\left(N-N_{*}\right)\left(f(N)-f\left(N_{*}\right)\right)}{f(N)} .
\end{aligned}
$$

A discussion similar to the proof of Theorem 1 shows that $\left(\mathrm{d} V_{2} / \mathrm{d} t\right) \leq 0$ and $\left(\mathrm{d} V_{2} / \mathrm{d} t\right)=0$ if and only if $(N, P)=\left(N_{*}\right.$, $\left.P_{*}\right)$. Hence, the properties of Lyapunov functional are satisfied, which indicates that the positive equilibrium $E_{*}$ is globally asymptotically stable when it exists. Considering the local asymptotical stability of the positive equilibrium, we obtain Theorem 4. This ends the proof.

Remark 1 . Theorems 2, 3, and 4 indicate that the local stability and global stability of the $E_{*}$ (or $E_{1}$ ) are equivalent, but the existence and stability of positive equilibrium $E_{*}$ and the local or global stability of boundary equilibrium $E_{1}$ are opposite.

\section{Dynamics of the Stochastic Model (3)}

In this section, we study the existence and uniqueness of the solution, stochastic extinction, persistence in the mean, and a unique ergodic stationary distribution of model (3). First of all, we define a bounded set $\Theta$ as follows:

$$
\Theta=\left\{(N, P) \in R_{+}^{2}: N+Q(x) P \leq \frac{I}{e}\right\},
$$

which will be used for the proofs of the following results.

3.1. Existence and Uniqueness of the Positive Solution. In model (3), the coefficients do not satisfy the linear growth condition, though they are locally Lipschitz continuous. Hence, the solution of model (3) may explore at a finite time. In this section, following the research [65], we prove that model (3) has a unique global positive solution.

Theorem 5. For any initial value $\left(N_{0}, P_{0}\right) \in \Theta$, there is a unique positive solution $(N(t), P(t))$ of model (3) on $t \geq 0$, and the solution will remain in $R_{+}^{2}$ with probability one.

Proof. Let $\left(N_{0}, P_{0}\right) \in \Theta$, based on the nutrient and phytoplankton equations in model (3) and by virtue of the positivity of $N$ and $P$, we can get 


$$
\begin{aligned}
\frac{\mathrm{d}(N+\mathrm{Q}(x) P)}{\mathrm{d} t} & =I-m N-(s(x)+d) Q(x) P \\
& \leq I-e(N+Q(x) P),
\end{aligned}
$$

then $\lim _{n \longrightarrow \infty} \sup (N+Q(x) P) \leq(I / e)$, that is,

$$
\begin{aligned}
& \lim _{t \rightarrow \infty} \sup N(t) \leq \frac{I}{e}, \\
& \lim _{t \rightarrow \infty} \sup P(t) \leq \frac{I}{e \beta x^{3}} .
\end{aligned}
$$

So, for any $c \in[0, t]$, we can obtain

$$
N(c), P(c) \in\left(0, \frac{I}{e}\right) \quad \text { a.s. }
$$

Consequently, the set $\Theta$ is a positivity invariant to model (3).

Since the coefficients of model (3) are locally Lipschitz continuous, for any given initial value $\left(N_{0}, P_{0}\right)$, there is a unique local solution $(N(t), P(t))$ on $t \in\left[0, \tau_{e}\right)$, where $\tau_{e}$ is the explosion time [67]. To show the solution is global, we need to show that $\tau_{e}=\infty$.

Let $n_{0}>0$ be sufficiently large for initial values $N_{0}$ and $P_{0}$ lying within the interval $\left[1 / n_{0}, n_{0}\right]$. For each integer $n>n_{0}$, considering the stopping times,

$$
\tau_{n}=\inf \left\{t \in\left[0, \tau_{n}\right), N(t) \notin\left(\frac{1}{n}, n\right) \text { or } P(t) \notin\left(\frac{1}{n}, n\right)\right\},
$$

and we set inf $\Phi=\infty$ ( $\Phi$ denotes the empty set). Obviously, $\tau_{n}$ is increasing as $n \longrightarrow \infty$. Let $\tau_{\infty}=\lim _{n \longrightarrow \infty} \tau_{n}$; hence, $\tau_{\infty} \leq \tau_{e}$ a.s. Next, we only need to show $\tau_{\infty}=\infty$. If this is false, there is a pair of constants $T>0$ and $\varepsilon \in(0,1)$ such that

$$
\mathbb{P}\left\{\tau_{n} \leq T\right\}>\varepsilon, n \geq n_{1} .
$$

Define a $C^{2}$ - function $V: R_{+}^{2} \longrightarrow R_{+}$by

$$
V(N, P)=-\ln \left(\frac{e N}{I}\right)-\ln \left(\frac{\beta x^{3} e P}{I}\right) .
$$

Using Itồ's formula [68], we have

$$
\begin{aligned}
\mathrm{d} V= & {\left[-\frac{I}{N}+\frac{\mu(x) Q(x) P}{a+N}+m+\frac{\delta^{2} \mu(x)^{2} \mathrm{Q}(x)^{2} P^{2}}{2(a+N)^{2}}\right.} \\
& \left.-\frac{\mu(x) N}{a+N}+s(x)+d+\frac{\delta^{2} \mu(x)^{2} N^{2}}{2(a+N)^{2}}\right] \mathrm{d} t \\
& +\frac{\delta \mu(x)(P Q(x)-N)}{a+N} \mathrm{~d} B \\
\leq & {\left[\frac{\mu(x) Q(x) P}{a}+m+\frac{\delta^{2} \mu(x)^{2} Q(x)^{2} P^{2}}{2 a^{2}}+s(x)+d\right.} \\
& \left.+\frac{\delta^{2} \mu(x)^{2} N^{2}}{2 a^{2}}\right] \mathrm{d} t+\frac{\delta \mu(x)(P Q(x)-N)}{a+N} \mathrm{~d} B \\
\leq & {\left[\frac{\mu(x) I}{a e}+m+s(x)+d+\frac{\delta^{2} \mu(x)^{2} I^{2}}{a^{2} e^{2}}\right] \mathrm{d} t } \\
& +\frac{\delta \mu(x)(P Q(x)-N)}{a+N} \mathrm{~d} B .
\end{aligned}
$$

Therefore,

$$
\mathrm{d} V(N(t), P(t)) \leq \varphi \mathrm{d} t+\frac{\delta \mu(x)(P Q(x)-N)}{a+N} \mathrm{~d} B
$$

where $\varphi=(\mu(x) I / a e)+m+s(x)+d+\left(\delta^{2} \mu(x)^{2} I^{2} / a^{2} e^{2}\right)$. Then,

$$
\begin{aligned}
\int_{0}^{\tau_{n} \wedge T} \mathrm{~d} V(N(t), P(t)) \leq & \int_{0}^{\tau_{n} \wedge T} \varphi \mathrm{d} t \\
& +\int_{0}^{\tau_{n} \wedge T} \frac{\delta \mu(x)(P Q(x)-N)}{a+N} \mathrm{~d} B
\end{aligned}
$$

where $\tau_{n} \wedge T=\min \left\{\tau_{n}, T\right\}$. Taking the expectation of the above inequality, we get

$$
\begin{aligned}
E V\left(N\left(\tau_{n} \wedge T\right), P\left(\tau_{n} \wedge T\right)\right) & \leq V\left(N_{0}, P_{0}\right)+\varphi E\left(\tau_{n} \wedge T\right) \\
& \leq V\left(N_{0}, P_{0}\right)+\varphi T
\end{aligned}
$$

Let $\Omega_{n}=\tau_{n} \leq T$ for $n \geq n_{1}$, by means of (23), then we have $\mathbb{P}\left(\Omega_{n}\right) \geq \mathcal{E}$. Note that, for every $\vartheta \in \Omega_{n}$, there is at least one of $N\left(\tau_{n}, \vartheta\right)$ and $P\left(\tau_{n}, \vartheta\right)$ that equals either $1 / n$ or $n$. Therefore, $V\left(N\left(\tau_{n}, \vartheta\right), P\left(\tau_{n}, \vartheta\right)\right)$ is not less than

$$
\begin{aligned}
\phi= & \min \left[-\ln \left(\frac{e n}{I}\right)-\ln \left(\frac{\beta e x^{3} n}{I}\right),-\ln \left(\frac{e n}{I}\right)-\ln \left(\frac{\beta e x^{3}}{I n}\right),\right. \\
& \left.-\ln \left(\frac{e}{n I}\right)-\ln \left(\frac{\beta e x^{3} n}{I}\right),-\ln \left(\frac{e}{n I}\right)-\ln \left(\frac{\beta e x^{3}}{n I}\right)\right] .
\end{aligned}
$$

It then follows from (28) that

$$
V\left(N_{0}, P_{0}\right)+\varphi T \geq E\left[1_{\Omega_{n}(\vartheta)} V(N(t), P(t))\right] \geq \varepsilon \phi,
$$

where $1_{\Omega_{n}(9)}$ is the indicator function of $\Omega_{n}$. Letting $n \longrightarrow \infty$, we have

$$
\infty>V\left(N_{0}, P_{0}\right)+\varphi T=\infty,
$$

which is a contradiction. Hence, we have $\tau_{\infty}=\infty$. That is, the solution of model (3) will not explore at a finite time with probability one. This completes the proof.

3.2. Stochastic Persistence and Extinction of Phytoplankton. From the proof of Theorem 5 , we know that $\Theta$ is the positive invariant set of model (3). In this section, we always assume that the initial value $\left(N_{0}, P_{0}\right) \in \Theta$ and first provide the result concerning the persistence of phytoplankton. For the sake of simplicity, we define

$$
\Lambda=\frac{\delta^{2} \mu^{2}(x) I^{2}}{2(e a+I)^{2}}+s(x)+d .
$$

Then, we have the following result.

Theorem 6. For any given initial $\left(N_{0}, P_{0}\right) \in \Theta$, if one of the following conditions holds: 


$$
\begin{aligned}
\text { (i) } \delta^{2} & <\frac{2 e(I+e a)}{\operatorname{Im} \mu(x)}, \\
X & >\frac{2 m(I+e a)^{2}(\mu(x)-s(x)-d)}{2 e I \mu(x)(I+e a)-I^{2} \delta^{2} m \mu^{2}(x)}, \\
\text { (ii) } \delta^{2} & >\frac{2 e(I+e a)}{\operatorname{Im} \mu(x)}, \\
X & <\frac{2 m(I+e a)^{2}(\mu(x)-s(x)-d)}{2 e I \mu(x)(I+e a)-I^{2} \delta^{2} \mu^{2}(x) m},
\end{aligned}
$$

then the solution of model (3) obeys

$$
\lim _{t \longrightarrow \infty} \inf \frac{1}{t} \int_{0}^{t} P(s) \mathrm{d} s \geq \frac{e I \mu(x)-\Lambda m(e a+I)}{e Q(x) \mu(x)(s(x)+d)}>0 \quad \text { a.s. }
$$

that is, the phytoplankton in model (3) is persistent in the mean almost surely.

Proof. Based on the nutrient and phytoplankton equations in model (3), we have

$$
d(N+\theta P)=(I-m N-(s(x)+d) Q(x) P) \mathrm{d} t .
$$

Integrating equation (35) from 0 to $t$ on the both sides, we get

$$
\begin{aligned}
(N(t)+Q(x) P(t))-\left(N_{0}+Q(x) P_{0}\right)= & t I-m \int_{0}^{t} N(s) \mathrm{d} s \\
& -(s(x)+d) Q(x) \\
& \cdot \int_{0}^{t} P(s) \mathrm{d} s .
\end{aligned}
$$

Note that $N(t)+Q(x) P(t)=N_{0}+Q(x) P_{0}$ for all $t>0$, and therefore, we adopt

$$
\frac{1}{t} \int_{0}^{t} N(s) \mathrm{d} s=\frac{I}{m}-\frac{(s(x)+d) Q(x)}{m} \frac{1}{t} \int_{0}^{t} P(s) \mathrm{d} s .
$$

By It $\widehat{o}$ 's formula, we have

$$
\begin{aligned}
d \ln P(t)= & {\left[-\frac{\delta^{2} \mu^{2}(x)}{2}\left(\frac{N}{a+N}\right)^{2}+\frac{\mu(x) N}{a+N}-s(x)-d\right] \mathrm{d} t } \\
& +\frac{\delta \mu(x) N}{a+N} \mathrm{~d} B .
\end{aligned}
$$

Let $f(N)=(N /(a+N))$, then it is easy to get $(\mathrm{d} f(N) / \mathrm{d} N)>0$. Hence, $f(N)$ is a strictly increasing function for any $N \in \mathrm{R}^{+}$. In light of $N \leq(I / e)$, we can obtain

$$
\begin{aligned}
-\frac{\delta^{2} \mu^{2}(x)}{2}\left(\frac{N}{a+N}\right)^{2}+\frac{\mu(x) N}{a+N}-s(x)-d \geq & -\frac{\delta^{2} \mu^{2}(x) I^{2}}{2(e a+I)^{2}} \\
& +\frac{\mu(x) e N}{e a+I}-s(x)-d .
\end{aligned}
$$

Integrating equation (38) from 0 to $t$ on the both sides, we have

$$
\begin{aligned}
\ln P(t)-\ln P(0) \geq & -\left[\frac{\delta^{2} \mu^{2}(x) I^{2}}{2(e a+I)^{2}}+s(x)+d\right] t \\
& +\frac{\mu(x) e}{e a+I} \int_{0}^{t} N(s) \mathrm{d} s+\kappa(t),
\end{aligned}
$$

where

$$
\kappa(t)=\int_{0}^{t} \frac{\delta \mu(x) N(s)}{a+N(s)} \mathrm{d} B(s) .
$$

Dividing $t$ on both sides of equation (40) and together with equation (37), we get

$$
\begin{aligned}
\frac{\ln P(t)}{t} \geq & \left(\frac{e I \mu(x)}{(e a+I) m}-\Lambda\right)-\frac{e Q(x) \mu(x)(s(x)+d)}{(e a+I) m} \frac{1}{t} \\
& \int_{0}^{t} P(s) \mathrm{d} s+\frac{\kappa(t)+\ln P(0)}{t} .
\end{aligned}
$$

Notice that $\kappa(t)$ is a local continuous martingale with $\kappa(0)=0$ and

$$
\lim _{t \longrightarrow \infty} \frac{\langle\kappa, \kappa\rangle_{t}}{t} \leq\left(\frac{\delta \mu(x) I}{a e+I}\right)^{2}<\infty .
$$

By the strong law of large number [69], we can achieve

$$
\lim _{t \rightarrow \infty} \frac{\kappa(t)+\ln P(0)}{t}=0 \quad \text { a.s. }
$$

By means of Lemma 4 in [70], if $\Lambda<(e I \mu(x) /(e a+I) m)$, that is,

$$
\begin{aligned}
2 m(I+e a)^{2}(\mu(x)-s(x)-d)< & X[2 e I \mu(x)(I+e a) \\
& \left.-I^{2} \delta^{2} \mu^{2}(x) m\right],
\end{aligned}
$$

which is equivalent to

$$
\begin{aligned}
X> & \frac{2 m(I+e a)^{2}(\mu(x)-s(x)-d)}{2 e I \mu(x)(I+e a)-I^{2} \delta^{2} m \mu^{2}(x)} \text { provided } \delta^{2} \\
& <\frac{2 e(I+e a)}{\operatorname{Im} \mu(x)}, \\
\text { or } X< & \frac{2 m(I+e a)^{2}(\mu(x)-s(x)-d)}{2 e I \mu(x)(I+e a)-I^{2} \delta^{2} \mu^{2}(x) m} \text { provided } \delta^{2} \\
& >\frac{2 e(I+e a)}{\operatorname{Im} \mu(x)},
\end{aligned}
$$

then it follows equation (42) that

$$
\lim _{t \longrightarrow \infty} \inf \frac{1}{t} \int_{0}^{t} P(s) \mathrm{d} s \geq \frac{e I \mu(x)-\Lambda m(I+e a)}{e Q(x) \mu(x)(s(x)+d)} \text { a.s. }
$$

This completes the proof.

Next, we study the stochastic extinction of phytoplankton in model (3). 
Theorem 7. For any given initial $\left(N_{0}, P_{0}\right) \in \Theta$, if one of the following conditions holds:

$$
\begin{aligned}
\text { (i) } \delta^{2} & <\frac{2(I+e a)}{I \mu(x)}, \\
X & <\frac{2(I+e a)^{2}(\mu(x)-s(x)-d)}{2 I \mu(x)(I+e a)-\delta^{2} \mu^{2}(x) I^{2}}, \\
\text { (ii) } \delta^{2} & >\frac{2(I+e a)}{I \mu(x)}, \\
X & >\frac{2(I+e a)^{2}(\mu(x)-s(x)-d)}{2 I \mu(x)(I+e a)-\delta^{2} I^{2} \mu^{2}(x)},
\end{aligned}
$$

then the solution of model (3) obeys

$$
\begin{gathered}
\lim _{t \rightarrow \infty} \sup \frac{\ln P(t)}{t} \leq \frac{I \mu(x)}{I+e a}-\Lambda<0 \quad \text { a.s., } \\
\text { or } \lim _{t \rightarrow \infty} \sup \frac{\ln P(t)}{t} \leq \frac{1}{2 \delta^{2}}-s(x)-d<0 \quad \text { a.s., }
\end{gathered}
$$

provided $\delta^{2}>\max \{(I+e a) / I \mu(x), 1 /(2(s(x)+d))\}$, which implies that the phytoplankton in model (3) is stochastic extinction with probability one.

Proof. Define the following quadratic function

$$
g(v)=-\frac{\delta^{2} \mu^{2}(x)}{2} v^{2}+\mu(x) v-s(x)-d,
$$

if the symmetric axis $1 / \delta^{2} \mu(x)$ of the equation $g(v)$ is larger than $I /(a e+I)$, that is, $\delta^{2} \leq((I+e a) / \mu(x) I)$, we can find that $g(v)$ takes its maximum value $g_{\max }$ on the interval $[0, I /(a e+I)]$ at $v=I /(a e+I)$, where

$$
g_{\max }=\frac{I \mu(x)}{I+e a}-\Lambda \text {. }
$$

Note that $f(N)=N /(a+N)$ is a strictly increasing function for $N \in[0, I / e]$ when $\delta^{2} \leq((I+e a) / I \mu(x))$; therefore, we have

$$
-\frac{\delta^{2} \mu^{2}(x)}{2}\left(\frac{N}{a+N}\right)^{2}+\frac{\mu(x) N}{a+N}-s(x)-d \leq g_{\max } .
$$

Integrating equation (38) from 0 to $t$ and dividing by $t$ on the both sides, we get

$$
\frac{\ln P(t)}{t} \leq\left(\frac{I \mu(x)}{I+e a}-\Lambda\right) t+\frac{\kappa(t)+\ln P(0)}{t} .
$$

Taking the limit of equation (53) and considering (44), we have

$$
\lim _{t \rightarrow \infty} \sup \frac{\ln P(t)}{t} \leq \frac{I \mu(x)}{I+e a}-\Lambda<0 \quad \text { a.s. }
$$

provided $\Lambda>(I \mu(x) /(I+e a))$, i.e.,

$$
\begin{aligned}
2(I+e a)^{2}(\mu(x)-s(x)-d)> & X[2 I \mu(x)(I+e a) \\
& \left.-\delta^{2} I^{2} \mu^{2}(x)\right],
\end{aligned}
$$

which equals to

$$
\begin{aligned}
\delta^{2} & <\frac{2(I+e a)}{I \mu(x)}, \\
X & <\frac{2(I+e a)^{2}(\mu(x)-s(x)-d)}{2 I \mu(x)(I+e a)-\delta^{2} \mu^{2}(x) I^{2}}, \\
\text { or } \delta^{2} & >\frac{2(I+e a)}{I \mu(x)}, \\
X & >\frac{2(I+e a)^{2}(\mu(x)-s(x)-d)}{2 I \mu(x)(I+e a)-\delta^{2} I^{2} \mu^{2}(x)} .
\end{aligned}
$$

On the other hand, if the symmetric axis $1 / \delta^{2} \mu(x)$ of the equation $g(v)$ is in the interval $[0, I /(e a+I)]$, i.e., $\delta^{2} \geq((I+e a) / I \mu(x))$, then the equation $g(v)$ reaches its maximum value $\left(1 / 2 \delta^{2}\right)-s(x)-d$ at $v=2 / \delta^{2} \mu(x)$. By performing a similar proof processes as above, we have

$$
\lim _{t \rightarrow \infty} \sup \frac{\ln P(t)}{t} \leq \frac{1}{2 \delta^{2}}-s(x)-d<0 \quad \text { a.s. }
$$

provided $\delta^{2}>\max \{(I+e a) / I \mu(x), 1 /(2(s(x)+d))\}$. This completes the proof.

Remark 2. Theorem 7 indicates that the phytoplankton in model (3) goes to extinction with probability one under some parameter conditions. In this case, it is natural to ask the question how will the nutrient concentration in model (3) change when the phytoplankton is extinct? In fact, when $\mathbb{P}\left\{\lim _{t \rightarrow \infty} P(t)=0\right\}=1$, then from the model (3), we can obtain the following equation:

$$
\mathrm{d} N(t)=(I-m N(t)) \mathrm{d} t
$$

by direct computation, we have $\lim _{t \longrightarrow \infty} N(t)=I / m$. Hence, the nutrient concentration will be a positive constant. That is, in this situation, the noise intensity and cell size will have no impacts on the variation of nutrient, and the dynamics of $N(t)$ in model (3) will be similar to the dynamics of $E_{1}$ in the deterministic model (1).

3.3. Existence of Ergodic Stationary Distribution. In this section, we shall establish sufficient conditions for the existence and uniqueness of an ergodic stationary distribution for model (3).

Let $Z(t)$ be a regular time-homogeneous Markov process in $R^{d}$ described by the stochastic differential equation:

$$
\mathrm{d} Z(t)=f(Z(t)) \mathrm{d} t+\sum_{l=1}^{k} g_{l}(Z) \mathrm{d} B_{l}(t) \mathrm{d} B(t) .
$$

The diffusion matrix of the process $Z(t)$ is defined as follows:

$$
\begin{aligned}
A(z) & =\left(a_{i j}(z)\right), \\
a_{i j}(z) & =\sum_{l=1}^{k} g_{l}^{i}(z) g_{l}^{j}(z) .
\end{aligned}
$$

Lemma 2 (see [71]). The Markov process $Z(t)$ has a unique ergodic stationary distribution $\mu(\cdot)$ if there exists a bounded open domain $D \subset R^{d}$ with regular boundary $\Gamma$, having the following properties: 
(B.1): there is a positive number $M$ such that $\sum_{i, j=1}^{d} a_{i, j}(z) \xi_{i} \xi_{j} \geq M|\xi|^{2}, z \in D$ and $\xi \in R^{d}$

(B.2): there exists a nonnegative $C^{2}$-function $V$ such that $\mathrm{LV}$ is negative for any $R^{d} \backslash D$

Theorem 8. If $X<\left(e^{2}(I+e a)^{2} /\left(e^{2}(I+e a)^{2} I+\delta^{2} I^{4}\right)\right)$, then for any given initial $\left(N_{0}, P_{0}\right) \in \Theta$, model (3) has a unique stationary distribution $\mu(\cdot)$ and it has the ergodic property.

Proof. To prove Theorem 8, we only need to validate condition (B.1) and (B.2) in Lemma 2. We first prove the condition (B.1). The diffusion matrix of model (3) is given by

$$
\begin{aligned}
\sum_{i, j=1}^{2} a_{i j}(N, P) \xi_{i} \xi_{j} & =\left(-\frac{\delta \mu(x) Q(x) N P \xi_{1}}{a+N} \frac{\delta \mu(x) N P \xi_{2}}{a+N}\right)\left(\begin{array}{c}
-\frac{\delta \mu(x) Q(x) N P \xi_{1}}{a+N} \\
\frac{\delta \mu(x) N P \xi_{2}}{a+N}
\end{array}\right) \\
& =\frac{\delta^{2} \mu^{2}(x) Q^{2}(x) N^{2} P^{2}}{(a+N)^{2}} \xi_{1}^{2}+\frac{\delta^{2} \mu^{2}(x) N^{2} P^{2}}{(a+N)^{2}} \xi_{2}^{2} \\
& \geq M_{0}\|\xi\|, \quad \text { for any }(N, P) \in \bar{D}_{\sigma} \subset \Theta, \\
\xi & =\left(\xi_{1}, \xi_{2}\right) \in R_{+}^{2},
\end{aligned}
$$

where $M_{0}=\min _{(N, P) \in \bar{D}_{\sigma}}\left\{\delta^{2} \mu^{2}(x) Q^{2}(x) N^{2} P^{2} /(a+N)^{2}, \delta^{2} \mu^{2}\right.$ $\left.(x) N^{2} P^{2} /(a+N)^{2}\right\}$, and then the condition (B.1) in Lemma 2 is satisfied. Next, we prove the condition (B.2).

Let

$$
M=\frac{2}{\lambda} \max \{2, H\},
$$

where $H=\sup _{(N, P) \in \Theta}\left\{-2 m N^{2}+2 I N-2 Q(x)(s(x)+d) P^{2}\right.$ $+\left(2 Q(x) I+e^{-1} \delta^{2} I \mu^{2}(x)(Q(x)+1)\right) P-2(s(x)+Q(x) m+$ d)PN\} and $\lambda=(1 / X)-\left(I+\left(\delta^{2} I^{4} / e^{2}(I+e a)^{2}\right)\right)>0$ provided $X<\left(e^{2}(I+e a)^{2} /\left(e^{2}(I+e a)^{2} I+\delta^{2} I^{4}\right)\right)$. Obviously, $(M \lambda / 4) \geq 1$. Then, we define a $C^{2}-$ function $V(N, P)$ : $\Theta \longrightarrow \mathrm{R}$ as follows:

$$
\begin{aligned}
V(N, P)= & M\left[N+P+\frac{N^{2}}{2 \mu^{2}(x)}+\frac{Q^{2}(x)}{\mu^{2}(x)} \frac{P^{2}}{2}\right. \\
& \left.+\frac{\ln P}{\mu(x)-s(x)-1}\right]+(N+Q(x) P)^{2},
\end{aligned}
$$

and it is easy to see that

$$
\lim _{n \longrightarrow+\infty,(N, P) \in \Theta \backslash D_{n}} V(N, P)=+\infty,
$$

where $D_{n}=(1 / n, n) \times(1 / n, n)$. Furthermore, $V(N, P)$ is continuous function, and so, $V(N, P)$ must have a minimum point $\left(N_{0}, P_{0}\right)$ in the interior of $\Theta$. Then, we define nonnegative $C^{2}-$ function $V(N, P): \Theta \longrightarrow R$ in the following form:

$$
\begin{aligned}
V(N, P)= & M\left[N+P+\frac{N^{2}}{2 \mu^{2}(x)}+\frac{Q^{2}(x)}{\mu^{2}(x)} \frac{P^{2}}{2}\right. \\
& \left.+\frac{\ln P}{\mu(x)-s(x)-1}\right]+(N+Q(x) P)^{2}-V\left(N_{0}, P_{0}\right) \\
:= & M\left[V_{1}(N, P)+V_{2}(N, P)+V_{3}(N, P)\right] \\
& +V_{4}(N, P),
\end{aligned}
$$

where $V_{1}(N, P)=N+P, V_{2}(N, P)=\left(N^{2} / 2 \mu^{2}(x)\right)+\left(Q^{2}\right.$ $\left.(x) / \mu^{2}(x)\right)\left(P^{2} / 2\right), \quad V_{3}=(\ln P /(\mu(x)-s(x)-1)), \quad$ and $V_{4}(N, P)=(N+Q(x) P)^{2}-V\left(N_{0}, P_{0}\right)$.

Making using of Itô's formula, we have

$$
\begin{aligned}
L V_{1}(N, P) & =I-\frac{\mu(x) Q(x) N P}{a+N}-m N+\frac{\mu(x) N P}{a+N}-(s(x)+d) P \\
& \leq I-m N-(s(x)+d) P+\frac{e \mu(x) N P}{I+e a} \\
& \leq I+\frac{e \mu(x) N P}{I+e a},
\end{aligned}
$$




$$
\begin{aligned}
L V_{2}(N, P)= & \frac{1}{\mu^{2}(x)}\left(I N-\frac{\mu(x) Q(x) P N^{2}}{a+N}-m N^{2}+\frac{\delta^{2} \mu^{2}(x) Q^{2}(x) N^{2} P^{2}}{2(a+N)^{2}}\right) \\
& +\left(\frac{Q(x)}{\mu(x)}\right)^{2}\left(\frac{\mu(x) N P^{2}}{a+N}-(s(x)+d) P^{2}+\frac{\delta^{2} \mu^{2}(x) N^{2} P^{2}}{2(a+N)^{2}}\right) \\
\leq & \frac{I N}{\mu^{2}(x)}-\frac{m N^{2}}{\mu^{2}(x)}+\frac{Q(x) N P}{\mu(x)(I+e a)}-\frac{(s(x)+d) Q^{2}(x) P^{2}}{\mu^{2}(x)}+\frac{\delta^{2} I^{4}}{e^{2}(I+e a)^{2}}, \\
L V_{3}(N, P)= & \frac{1}{\mu(x)-s(x)-1}\left(\frac{\mu(x) N}{a+N}-(s(x)+d)-\frac{\delta^{2} \mu^{2}(x) N^{2}}{2(a+N)^{2}}\right) \\
\leq & \frac{\mu(x) N}{a(\mu(x)-s(x)-d)}-\frac{1}{X}-\frac{\delta^{2} e^{2} N^{2} \mu^{2}(x)}{2(e a+I)^{2}(\mu(x)-s(x)-d)}, \\
L V_{4}(N, P)= & 2\left(N I-m N^{2}-(s(x)+d) N P+Q(x) I P-Q(x)(s(x)+d) P^{2}\right) \\
& +\frac{\delta^{2} \mu^{2}(x) Q(x)(Q(x)+1) N^{2} P^{2}}{(a+N)^{2}} \\
\leq & -2 m N^{2}+2 I N-2 Q(x)(s(x)+d) P^{2}+\left(2 Q(x) I+e^{-1} \delta^{2} I \mu^{2}(x)(Q(x)+1)\right) P \\
& -2(s(x)+Q(x) m+d) P N .
\end{aligned}
$$

Hence, we get

$$
\begin{aligned}
L V(N, P)= & M\left(L V_{1}(N, P)+L V_{2}(N, P)+L V_{3}(N, P)\right)+V_{4}(N, P) \\
\leq & -M \lambda+M\left[\left(\frac{I}{\mu^{2}(x)}+\frac{\mu(x)}{a(\mu(x)-s(x)-d)}\right) N+\frac{\left(Q(x)+e \mu^{2}(x)\right) N P}{\mu(x)(I+e a)}-\left(\frac{m}{\mu^{2}(x)}\right.\right. \\
& \left.\left.+\frac{\delta^{2} e^{2} \mu^{2}(x)}{2(e a+I)^{2}(\mu(x)-s(x)-d)}\right) N^{2}-\frac{(s(x)+d) Q^{2}(x)}{\mu^{2}(x)} P^{2}\right] \\
& +\left[-2 m N^{2}+2 I N-2 Q(x)(s(x)+d) P^{2}+\left(2 Q(x) I+e^{-1} \delta^{2} I \mu^{2}(x)(Q(x)+1)\right) P\right. \\
& -2(s(x)+Q(x) m+d) P N] .
\end{aligned}
$$

Define a bounded closed set

$$
D_{\varepsilon}=\left\{(N, P) \in \Theta: \varepsilon \leq N \leq \frac{1}{\varepsilon}, \varepsilon \leq P \leq \frac{1}{\varepsilon}\right\},
$$

where $0<\varepsilon<1$ is a sufficiently small number. We choose $\varepsilon$ sufficiently small such that

$$
\begin{aligned}
& 0<\varepsilon<\frac{\lambda}{4\left(\left(I / \mu^{2}(x)\right)+(\mu(x) / a(\mu(x)-s(x)-d))+\left(\left(Q(x)+e \mu^{2}(x)\right) / \mu(x)(I+e a)\right)\right)}, \\
& 0<\varepsilon<\frac{Q^{2}(x)(s(x)+d)(I+e a)}{\mu(x)\left(Q(x)+e \mu^{2}(x)\right)},
\end{aligned}
$$




$$
\begin{aligned}
0<\varepsilon<\frac{\lambda \mu(x)(I+e a)}{2\left(Q(x)+e \mu^{2}(x)\right)}, \\
0<\varepsilon<\frac{2 m(I+e a)^{2}(\mu(x)-s(x)-d)+\delta^{2} e^{2} \mu^{4}(x)}{2 \mu(x)(I+e a)(\mu(x)-s(x)-d)\left(Q(x)+e^{2} \mu(x)\right)}, \\
-\frac{M \lambda}{2}+\bar{H} \leq-1, \\
-M \lambda-\left(\frac{m M}{\mu^{2}(x)}+\frac{M \delta^{2} e^{2} \mu^{2}(x)}{2(e a+I)^{2}(\mu(x)-s(x)-d)}\right) \frac{1}{\varepsilon^{2}}+\bar{H}_{*}, \\
-M \lambda-M \frac{(s(x)+d) Q^{2}(x)}{\mu^{2}(x)} \frac{1}{\varepsilon^{2}}+\bar{H}_{*},
\end{aligned}
$$

where

$$
\begin{aligned}
\bar{H}= & \sup _{(N, P) \in \Theta}\left[-2 m N^{2}+2 I N-2 Q(x)(s(x)+d) P^{2}\right. \\
& +\left(2 Q(x) I+e^{-1} \delta^{2} I \mu^{2}(x)(Q(x)+1)\right) P-2(s(x) \\
& +Q(x) m+d) P N+M\left(\frac{I}{\mu^{2}(x)}\right. \\
& \left.\left.+\frac{\mu(x)}{a(\mu(x)-s(x)-d)}\right) N\right], \\
\bar{H}_{*}= & \sup _{(N, P) \in \Theta}\left[-2 m N^{2}+2 I N-2 Q(x)(s(x)+d) P^{2}\right. \\
& +\left(2 Q(x) I+e^{-1} \delta^{2} I \mu^{2}(x)(Q(x)+1)\right) P-2(s(x) \\
& +Q(x) m+d) P N+\left(\frac{I}{\mu^{2}(x)}+\frac{\mu(x)}{a(\mu(x)-s(x)-d)}\right) N \\
& \left.+\frac{\left.\left(Q(x)+e \mu^{2}(x)\right) N P\right]}{\mu(x)(I+e a)}\right] .
\end{aligned}
$$

For convenience, we divide $\Theta \backslash D_{\varepsilon}$ into four domains:

$$
\begin{aligned}
& D_{\varepsilon}^{1}=\{(N, P) \in \Theta: 0<N<\varepsilon\}, \\
& D_{\varepsilon}^{2}=\{(N, P) \in \Theta: 0<P<\varepsilon\}, \\
& D_{\varepsilon}^{3}=\left\{(N, P) \in \Theta: N>\frac{1}{\varepsilon}\right\}, \\
& D_{\varepsilon}^{4}=\left\{(N, P) \in \Theta: P>\frac{1}{\varepsilon}\right\} .
\end{aligned}
$$

Clearly, $D_{\varepsilon}^{C}=\cup_{i}^{4} D_{\varepsilon}^{i}$. Now, we prove that $L V(N, P) \leq 1$ on $D_{\varepsilon}^{C}$, which is equivalent to prove it on $D_{\varepsilon}^{1}, D_{\varepsilon}^{2}, D_{\varepsilon}^{3}$, and $D_{\varepsilon}^{4}$, respectively.

Case 1. When $(N, P) \in D_{\varepsilon}^{1}$, since $N P \leq \varepsilon P \leq \varepsilon\left(1+P^{2}\right)$, we have

$$
\begin{aligned}
L V(N, P) \leq & -\frac{M \lambda}{4}+\left[\left(\frac{M I \varepsilon}{\mu^{2}(x)}+\frac{M \mu(x) \varepsilon}{a(\mu(x)-s(x)-d)}\right.\right. \\
& \left.\left.+\frac{\left(Q(x)+e \mu^{2}(x)\right) M \varepsilon}{\mu(x)(I+e a)}\right)-\frac{M \lambda}{4}\right] \\
& -\left(\frac{m M}{\mu^{2}(x)}+\frac{M \delta^{2} e^{2} \mu^{2}(x)}{2(e a+I)^{2}(\mu(x)-s(x)-d)}\right) N^{2} \\
& +\left[\frac{\left(Q(x)+e \mu^{2}(x)\right) M \varepsilon}{\mu(x)(I+e a)}-\frac{M(s(x)+d) Q^{2}(x)}{\mu^{2}(x)}\right] P^{2} \\
& +\left[-\frac{M \lambda}{2}+H\right],
\end{aligned}
$$

which together with conditions (72) and (73), as well as the definition of $M$ in (62), yields

$$
L V(N, P) \leq-\frac{M \lambda}{4} \leq-1
$$

Case 2. When $(N, P) \in D_{\varepsilon}^{2}$, since $N P \leq \varepsilon N \leq \varepsilon\left(1+N^{2}\right)$, we get

$$
\begin{aligned}
L V(N, P) \leq & {\left[-\frac{M \lambda}{2}+\frac{\left(Q(x)+e \mu^{2}(x)\right) M \varepsilon}{\mu(x)(I+e a)}\right] } \\
& -\frac{M Q^{2}(x)(s(x)+d)}{\mu^{2}(x)} P^{2} \\
& +\left[\frac{M\left(Q(x)+e \mu^{2}(x)\right) \varepsilon}{\mu(x)(I+e a)}-M\left(\frac{m}{\mu^{2}(x)}\right.\right. \\
& \left.\left.+\frac{\delta^{2} e^{2} \mu^{2}(x)}{2(I+e a)^{2}(\mu(x)-s(x)-d)}\right)\right] N^{2} \\
& +\left[-\frac{M \lambda}{2}+\bar{H}\right],
\end{aligned}
$$

which leads to $L V(N, P) \leq-(M \lambda / 2)+\bar{H} \leq 1$ in this domain, in the view of (74)-(76). 
Case 3. When $(N, P) \in D_{\varepsilon}^{3}$, we have

$$
\begin{aligned}
L V(N, P) \leq & -M \lambda+M\left[-\left(\frac{m}{\mu^{2}(x)}\right.\right. \\
& \left.+\frac{\delta^{2} e^{2} \mu^{2}(x)}{2(e a+I)^{2}(\mu(x)-s(x)-d)}\right) N^{2} \\
& \left.-\frac{(s(x)+d) Q^{2}(x)}{\mu^{2}(x)} P^{2}\right]+\left[-2 m N^{2}+2 I N\right. \\
& -2 Q(x)(s(x)+d) P^{2}+\left(2 Q(x) I+e^{-1} \delta^{2} I \mu^{2}\right. \\
& \cdot(x)(Q(x)+1) P-2(s(x)+Q(x) m+d) P N \\
& +\left(\frac{I}{\mu^{2}(x)}+\frac{\mu(x)}{a(\mu(x)-s(x)-d)}\right) N \\
& \left.+\frac{\left.\left(Q(x)+e \mu^{2}(x)\right) N P\right]}{\mu(x)(I+e a)}\right] \\
\leq & -M \lambda-\left(\frac{m M}{\mu^{2}(x)}\right) N^{2}+\bar{H}_{*}, \\
& =\frac{M \delta^{2} e^{2} \mu^{2}(x)}{2(e a+I)^{2}(\mu(x)-s(x)-d)}
\end{aligned}
$$

from (77), and we have $L V(N, P) \leq-1$ on $(N, P) \in D_{\varepsilon}^{3}$.

Case 4. When $(N, P) \in D_{\varepsilon}^{4}$, we have

$$
\begin{aligned}
L V(N, P) \leq & -M \lambda+M\left[-\left(\frac{m}{\mu^{2}(x)}\right.\right. \\
& \left.+\frac{\delta^{2} e^{2} \mu^{2}(x)}{2(e a+I)^{2}(\mu(x)-s(x)-d)}\right) N^{2} \\
& \left.-\frac{(s(x)+d) Q^{2}(x)}{\mu^{2}(x)} P^{2}\right]+\left[-2 m N^{2}+2 I N\right. \\
& -2 Q(x)(s(x)+d) P^{2}+\left(2 Q(x) I+e^{-1} \delta^{2} I \mu^{2}\right. \\
& \cdot(x)(Q(x)+1)) P-2(s(x)+Q(x) m+d) P N \\
& +\left(\frac{I}{\mu^{2}(x)}+\frac{\mu(x)}{a(\mu(x)-s(x)-d)}\right) N \\
& \left.+\frac{\left.\left(Q(x)+e \mu^{2}(x)\right) N P\right]}{\mu(x)(I+e a)}\right] \\
\leq & -M \lambda-M \frac{(s(x)+d) Q^{2}(x)}{\mu^{2}(x)} P^{2}+\bar{H}_{*},
\end{aligned}
$$

in view of (78), and we get $L V(N, P) \leq-1$. This ends the proof.

\section{Numerical Results}

In this section, we study numerically the impact of the cell size effect on the nutrient-phytoplankton dynamics in the deterministic model (1) as well as the stochastic model (3). The parameter values are taken as shown in Table 1. Unless stated otherwise, we will use them for our simulations. Additionally, in the following numerical simulation, we randomly select the initial value satisfying $\Theta$.

4.1. Impact of Cell Size Factor in the Nutrient-Phytoplankton Dynamics of Model (1). In this section, we study the impact of the cell size effect on the concentration of nutrient and the density of phytoplankton, as well as the stability of the equilibria in model (1).

On the basis of previous theoretical analysis, let $f(x)=X-(m a / I)$, and substituting (4) and the required parameters into $f(x)$, we get $f(x)<0$, i.e., $X<(m a / I)$ if

$$
H_{1}:=\{x \in R \mid 0<x<0.07807216867 \text { or } 2.981219088<x\} \text {, }
$$

and $f(x)>0$, i.e., $X>(m a / I)$ if

$$
H_{2}:=\{x \in R \mid 0.07807216867<x<2.981219088\} .
$$

Next, we first show the impact of cell size on the nutrient concentration and phytoplankton density.

4.1.1. Impact of the Cell Size Effect on the Nutrient Concentration and Phytoplankton Density. Based on the analytical expressions for the positive equilibrium level of nutrient and phytoplankton, one can know that the nutrient concentration and phytoplankton density are closely related to cell size $x$. However, let $x \in H_{1}$; the increase of cell size $x$ does not affect the nutrient concentration, which is clearly demonstrated in Figures 1(a) and 1(c). Furthermore, in this case, Figures 1(d) and $1(\mathrm{f})$ show that the density of phytoplankton is negative or zero. That is, the phytoplankton in model (1) cannot survive when the phytoplankton cell is smaller or larger $\left(x \in H_{1}\right)$, which may be the main reason why the nutrient concentration does not change with the increase of cell size.

However, as the cell size increases to the domain of $\mathrm{H}_{2}$, the nutrient concentration is a nonmonotonic concave function with respect to cell size $x$ (see Figure 1(b)) and phytoplankton density is a nonmonotonic convex function of cell size $x$ (see Figure 1(e)). Nevertheless, by further analysis, we find that

(1) If $x \in(0.07807216867,0.1]$, the nutrient concentration is a monotonic decreasing function with respect to cell size $x$ (see Figure $2(\mathrm{a})$ ), but phytoplankton density is a convex-line increasing function of cell size $x$ (see Figure 2(d)), which signifies that phytoplankton can reproduce rapidly and abundantly and absorb a large amount of nutrients during its growth, resulting in a rapid increase in phytoplankton density and a corresponding rapid decrease in nutrient concentration. In fact, from 
TABle 1: Parameter values in numerical simulations for model (1) and model (3).

\begin{tabular}{lcc}
\hline Parameters & Unit & Values \\
\hline : the nutrient input rate & $\mu \mathrm{mol} / \mathrm{Lay}$ & $10[72]$ \\
$\alpha:$ the phytoplankton sinking rate coefficient & $1 /$ day $/ \mu \mathrm{m}^{2}$ & $0.1[55]$ \\
$\beta:$ the phytoplankton nutrient quota coefficient & $\mu \mathrm{mol}$ nutrient $/ \mathrm{cell}^{-b_{1}}$ & $100(\mathrm{estimate})$ \\
$m:$ the removal rate of nutrient & $1 /$ day & $0.85(\mathrm{estimate})$ \\
$d:$ the death rate of phytoplankton & $1 /$ day & $0.1[55]$ \\
$a:$ the half-saturation constant & $\mu \mathrm{mol} / L$ & $100(\mathrm{estimate})$ \\
$a_{1}:$ a positive empirical constant & $1 / \mu \mathrm{m}^{2}$ & $0.02[55]$ \\
$a_{2}:$ a positive empirical constant & $1 / \mu \mathrm{m}$ & $0.02[55]$ \\
$a_{3}:$ a positive empirical constant & Unitless & $0.08[55]$ \\
\hline
\end{tabular}

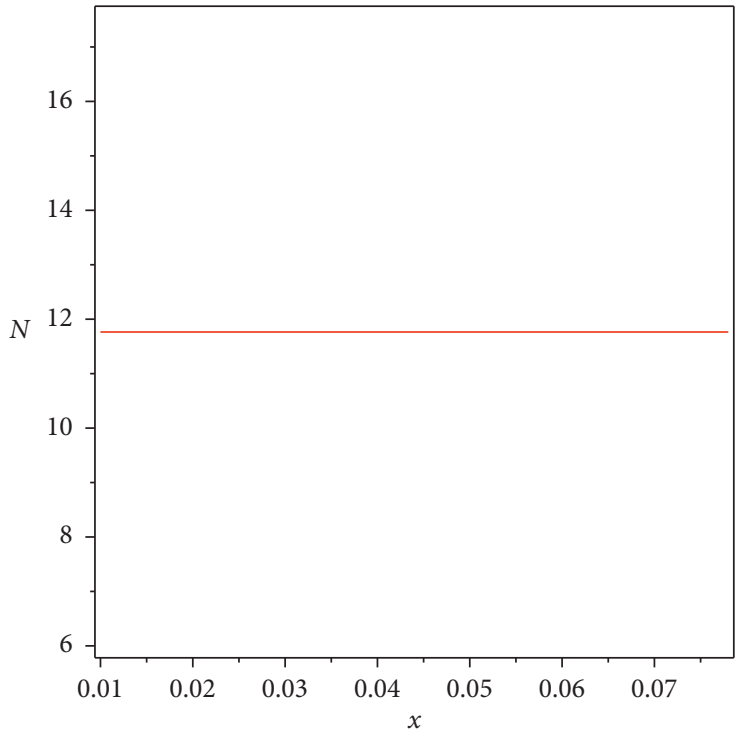

(a)

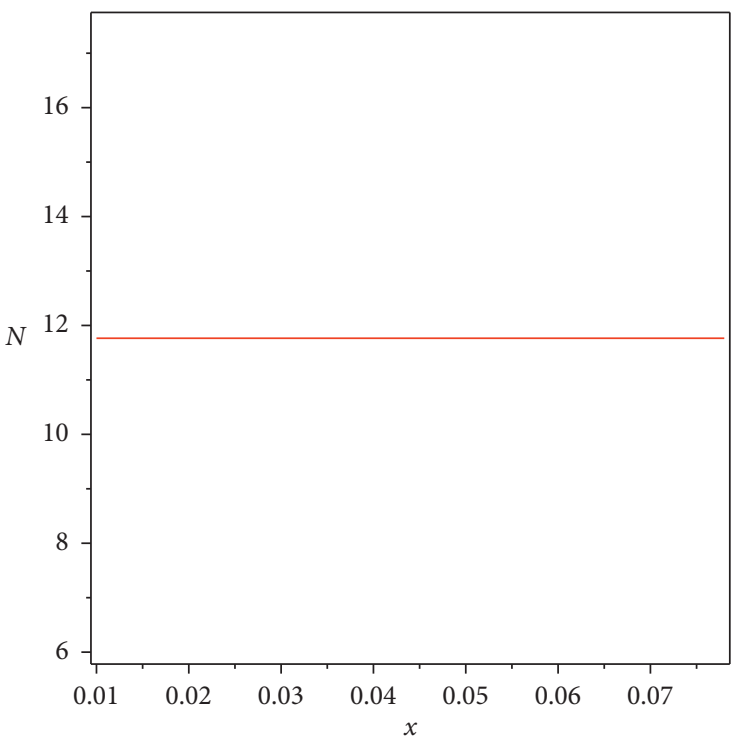

(c)

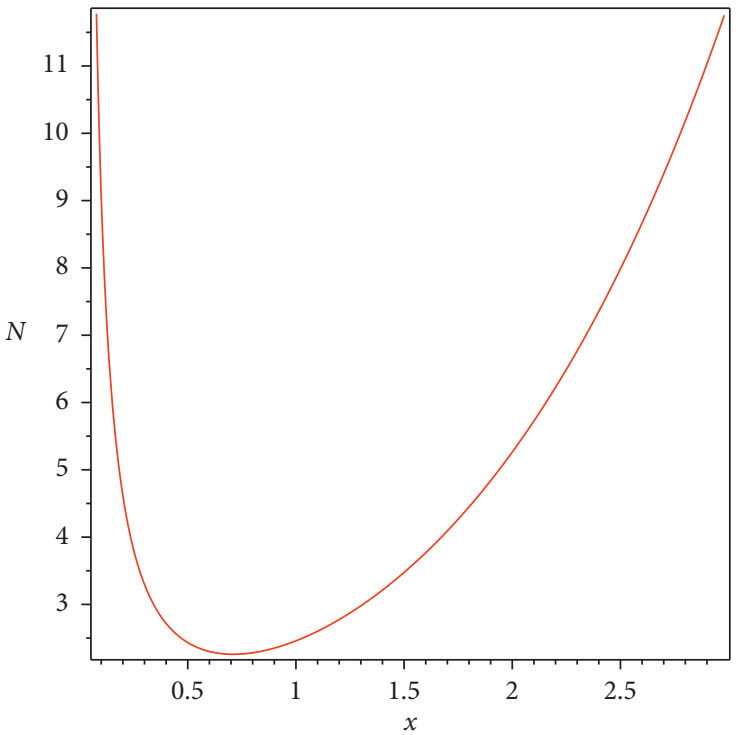

(b)

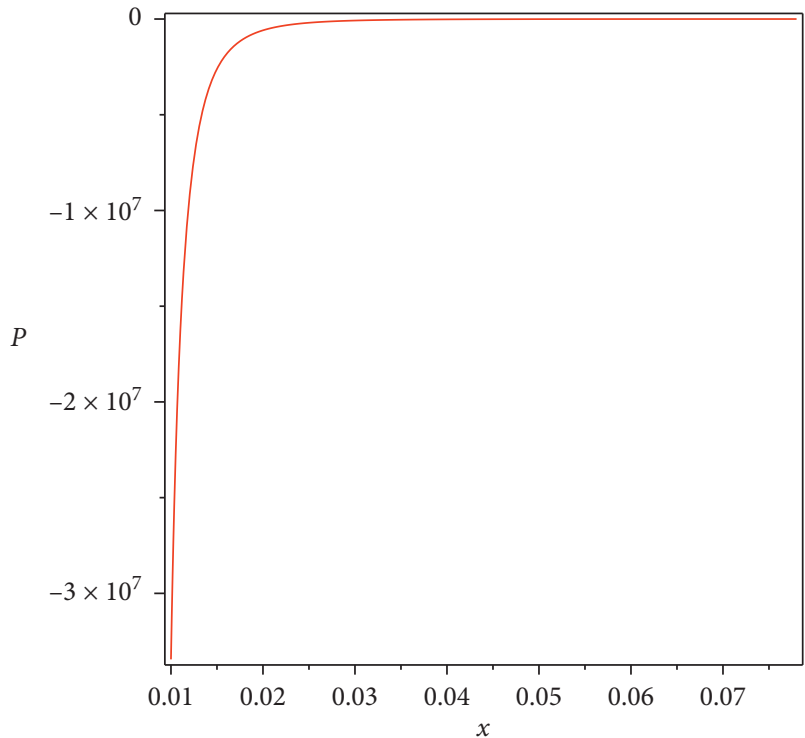

(d)

Figure 1: Continued. 


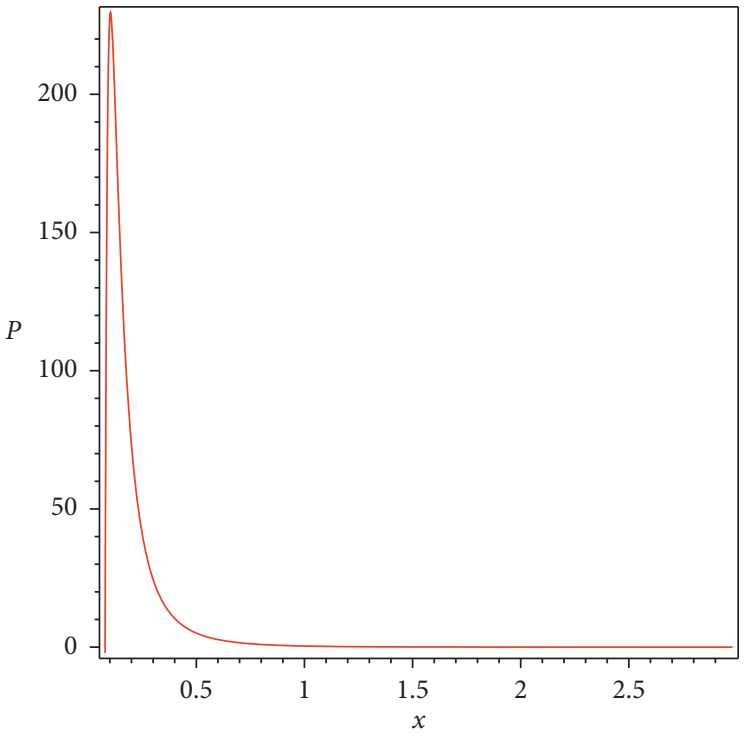

(e)

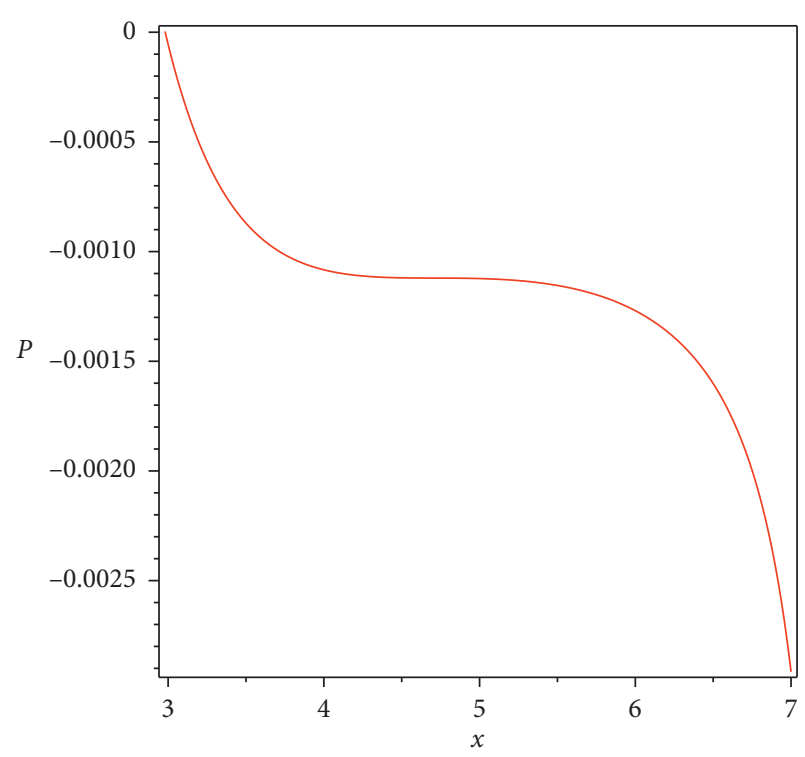

(f)

FIGURE 1: (a-c) Bifurcation diagram of nutrient in model (1) with respect to $x$. (d-f) Bifurcation diagram of phytoplankton in model (1) with respect to $x$. (a) and (d) for $x \in(0.01,0.07807216867)$. (b) and (e) for $x \in(0.07807216867,2.981219088)$. (c) and (f) for $x \in(2.981219088,7)$.

Figure 1(e) or Figures 2(d) and 2(e), we can know that the peak of phytoplankton density appears at $x=0.1$, and so, $x=0.1$ is the optimal size for promoting the rapid reproduction of the phytoplankton population.

(2) If $x \in(0.1,0.6]$, both the nutrient concentration and phytoplankton density are concave-line decreasing function of cell size $x$, which are shown in Figures 2(b) and 2(e), respectively. As the density of phytoplankton reaches its peak at $x=0.1$, when the cell size of phytoplankton exceeds its optimal size, the phytoplankton density decreases with the increase of cell size, but its density is still very high, resulting in the decrease of nutrient concentration, whereas the reduction rate of the nutrient concentration is decreasing compared with that in Figure 2(a).

(3) If $x \in(0.6,2.981219088)$, the nutrient concentration is a concave-line increasing function with respect to $x$ (see Figure 2(c)), but phytoplankton density is a concave-line decreasing function of cell size, as is shown in Figure 2(f). Obviously, in this case, with the increase of cell size, the density of phytoplankton decreases dramatically with the rapid increase of nutrient concentration.

4.1.2. Impact of Cell Size Effect on the Stability of the Equilibria. The analysis indicates that the plane is divided into two regions (i) and (ii) by $I-x$ parameters, as displayed in Figure 3(a). However, when the phytoplankton cell $x$ is smaller or larger $\left(x \in H_{1}\right)$, there exists a unique boundary equilibrium $E_{1}$ in the area (i), which is globally asymptotically stable (see Theorem 3 ). With the increase of cell size $\left(x \in H_{2}\right)$, the positive equilibrium $E_{*}$ appears in (ii) and it is globally asymptotically stable, but the $E_{1}$ becomes unstable (see Theorems 2 and 4). Then, we adopt $x=0.05 \in H_{1}$ and $x=0.51 \in \mathrm{H}_{2}$; the corresponding results concerning the global stability of $E_{1}=(11.76470588,0)$ and $E_{*}=(2.414081849,4.754928396)$ are shown in (i) and (ii) of Figure 3(b), respectively. It is worth noting that the only difference between (i) and (ii) in Figure 3(b) is that the cell size of phytoplankton is different. Actually, from the Figure 3(a), it is clear that the increase of cell size can lead to the stability changes at the boundary equilibrium $E_{1}$ : stable $(0<x<0.07807216867) \longrightarrow$ unstable $\quad(0.07807216867$ $<x<2.981219088) \longrightarrow$ stable $(x>2.981219088)$, and the existence of positive equilibrium $E_{*}$ changes: nonexistence $(0<x<0.07807216867) \longrightarrow$ existence $\quad(0.07807216867$ $<x<2.981219088) \longrightarrow$ nonexistence $(x>2.981219088)$, which indicates that the cell size plays an important role in determining the existence and stability of the equilibria in model (1).

4.2. Impact of Cell Size Factor in the Nutrient-Phytoplankton Dynamics of Model (3). Now, we turn to study the impact of cell size effect on the stochastic dynamics of model (3) by using the Milstein method mentioned in Higham [73]. In this way, model (3) can be rewritten as the following discretization equation: 


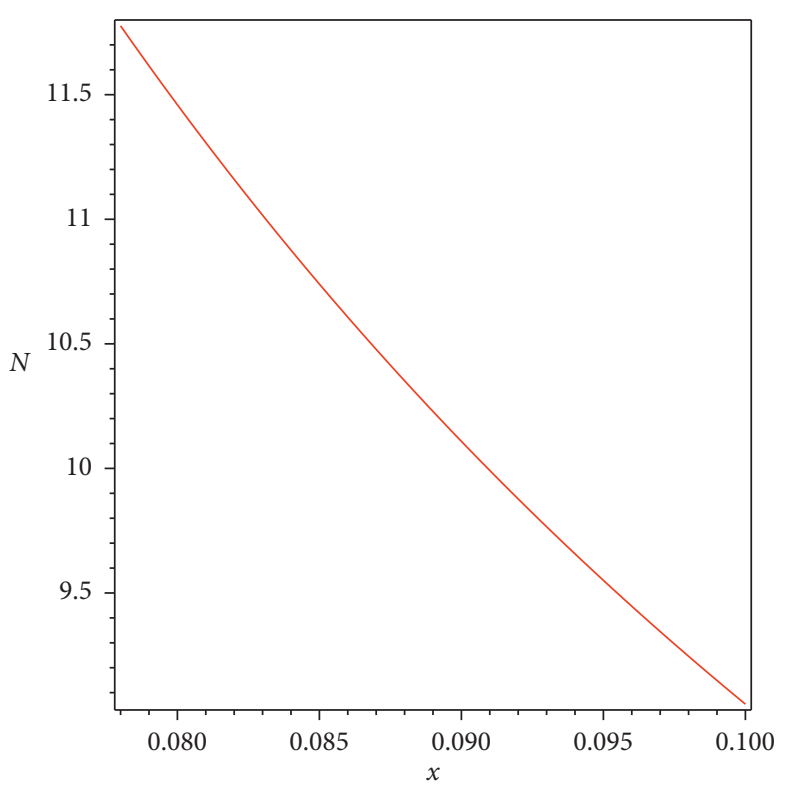

(a)

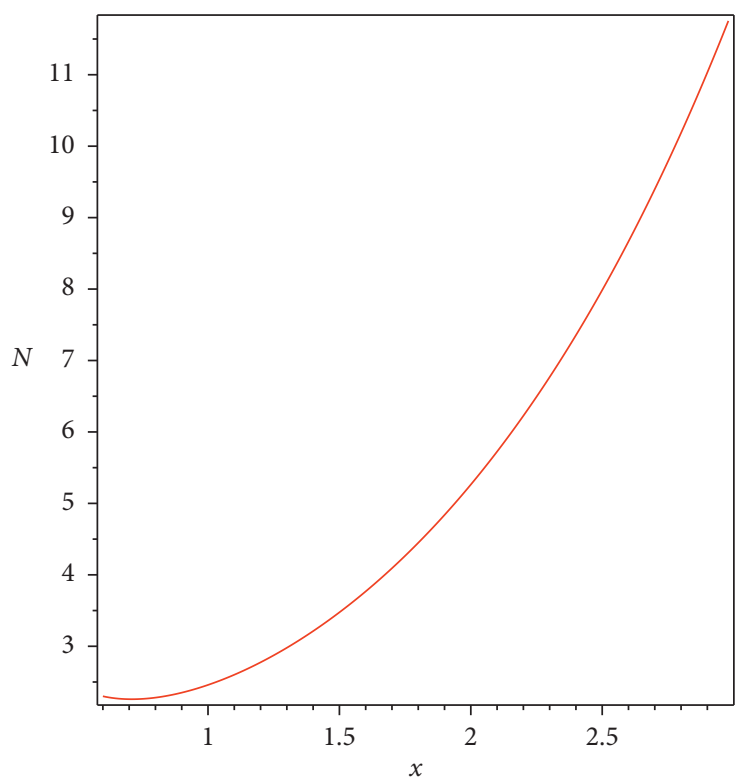

(c)

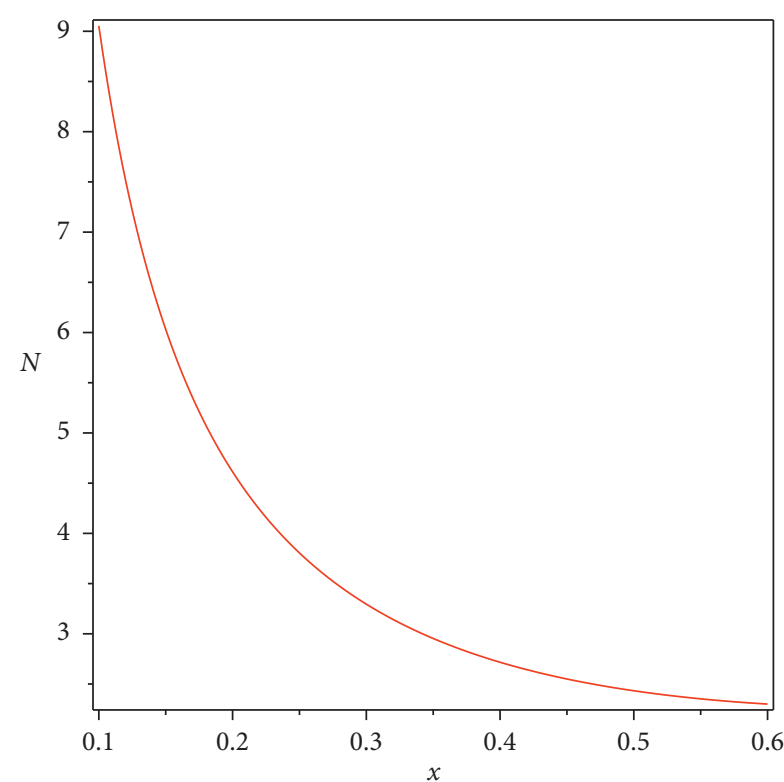

(b)

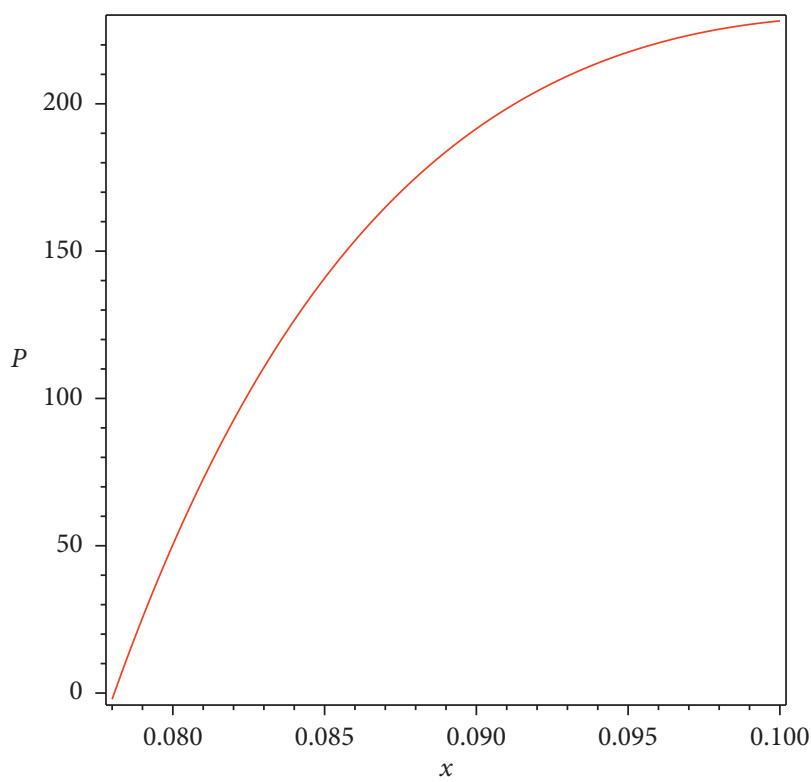

(d)

Figure 2: Continued. 


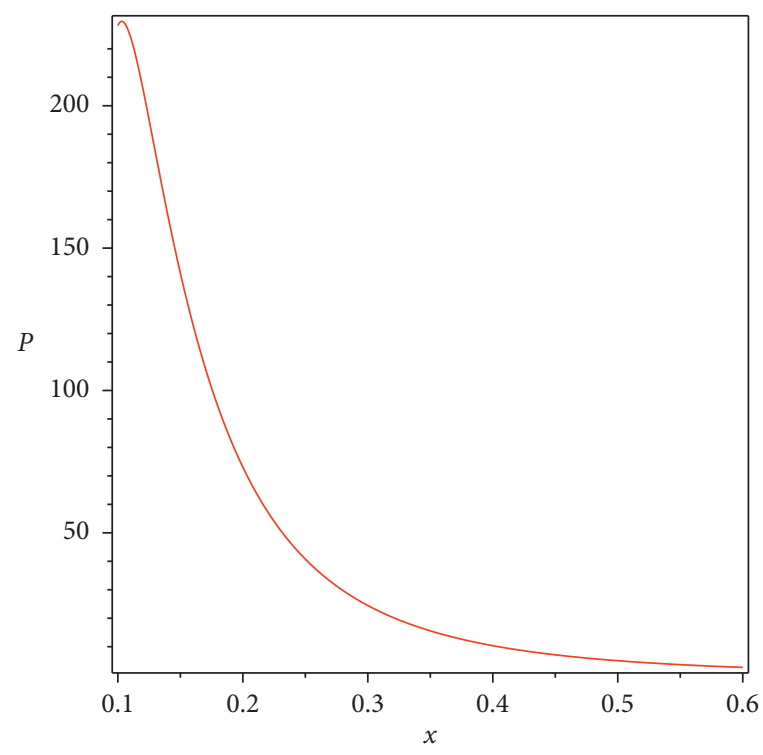

(e)

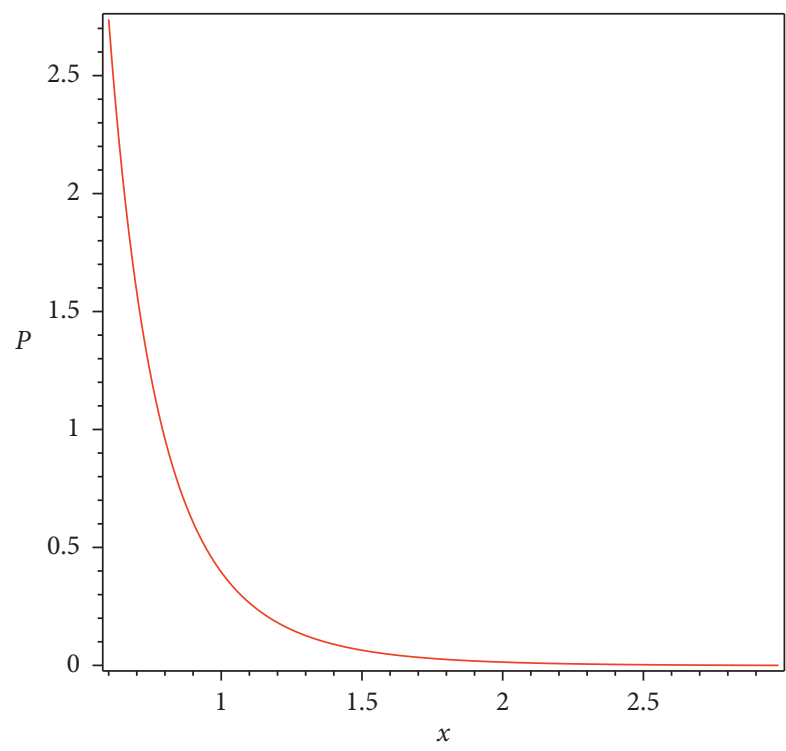

(f)

Figure 2: (a-c) Bifurcation diagram of nutrient in model (1) with respect to $x \in \mathrm{H}_{2}$. (b-f) Bifurcation diagram of phytoplankton in model (1) with respect to $x \in H_{2}$. (a) and (d) for $x \in(0.07807216867,0.1]$. (b) and (e) for $x \in(0.1,0.6]$. (c) and (f) for $x \in(0.6,2.981219088)$.

$$
\left\{\begin{array}{l}
N_{k+1}=N_{k}+\left(I-\frac{\mu(x) Q(x) N_{k} P_{k}}{a+N_{k}}-m N_{k}\right) \Delta t \\
-\frac{\delta \mu(x) Q(x) N_{k} P_{k}}{a+N_{k}} \sqrt{\Delta t} \xi_{k}+\frac{\delta^{2}}{2} \frac{\mu(x) Q(x) N_{k} P_{k}}{a+N_{k}}\left(\xi_{k}^{2}-1\right) \Delta t \\
P_{k+1}=P_{k}+\left(\frac{\mu(x) N_{k} P_{k}}{a+N_{k}}-s(x) P_{k}-d P_{k}\right) \Delta t \\
+\frac{\delta \mu(x) N_{k} P_{k}}{a+N_{k}} \sqrt{\Delta t} \xi_{k}+\frac{\delta^{2}}{2} \frac{\mu(x) N_{k} P_{k}}{a+N_{k}}\left(\xi_{k}^{2}-1\right) \Delta t
\end{array}\right.
$$

where $\xi_{k}(k=1,2, \ldots, n)$ are independent Gaussian random variables $N(0,1)$.

4.2.1. Impact of Cell Size and Environmental Noise Effects on the Survival of Phytoplankton. First of all, we fix $x=0.51$ and vary $\delta=0.1,1.8$ to investigate how environmental noise affects the persistence of model (3).

When we take $\delta=0.1$, by simple computations, it is not difficult to get that the condition (i) in Theorem 6 and the condition in Theorem 8 are satisfied. From Theorems 6 and 8 , it can be obtained that model ( 3 ) is persistent in the mean and exists a unique stationary distribution, as is shown in Figure 4. This result signifies that the small environmental noise disturbance can make model (3) maintain some stability in the random sense and drive the solutions to be perturbed near the positive equilibrium of the deterministic model (1).
When we obtain the noise intensity $\delta=1.8$, then the condition (ii) of Theorem 7 is satisfied. Based on Theorem 7 , the phytoplankton goes to extinction with probability one, which implies that the larger environmental noise can result in the extinction of phytoplankton. Figure 5(b) clearly confirms this result. Further, in this case, the nutrient concentration is found to increase to $N=N_{1}=$ $11.76470588>N_{*}=2.414081849$ (see Figure 5(a)), which agrees well with Remark 2.

Next, we fix $\delta=0.1$ and change $x=0.001,0.15$, $0.45,0.95$ to see the effect of cell size on the survival of phytoplankton in model (3). It is clear from Figure 6 that if $x=0.15$ or $0.45 \subset \mathrm{H}_{2}$, the phytoplankton is persistent and becomes extinct when $x=0.001$ or $0.98 \subset H_{1}$, which indicates that the smaller or larger cell size can result in the extinction of phytoplankton (see the black and pink curves) and the intermediate cell size is advantage for the persistence of phytoplankton (see the blue and green curves). Moreover, by comparing the green curve with the blue curve, it can be found that the increase of cell size can reduce the random variation of phytoplankton caused by environmental noise disturbance and decrease the density of phytoplankton.

4.2.2. Impact of Cell Size Effect on the Existence of Ergodic Stationary Distribution. In order to explore how cell size affects the existence of stationary distribution of phytoplankton and nutrient in model (3), we fix the noise intensity $\delta=0.1$ and vary the cell size

$$
x=0.01,0.02,0.03,0.04,0.05,0.06,
$$




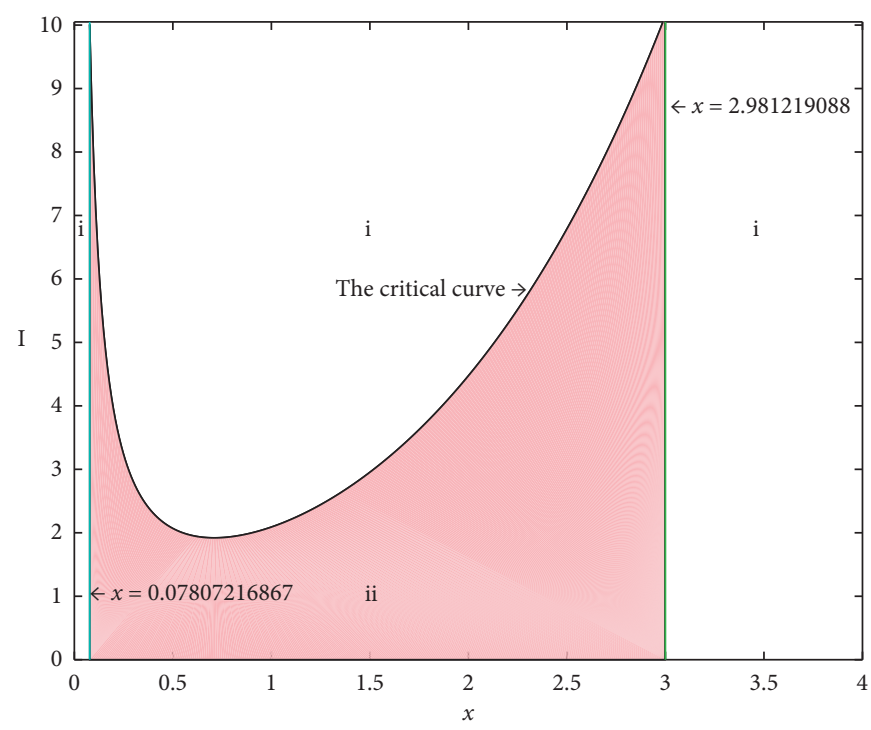

(a)

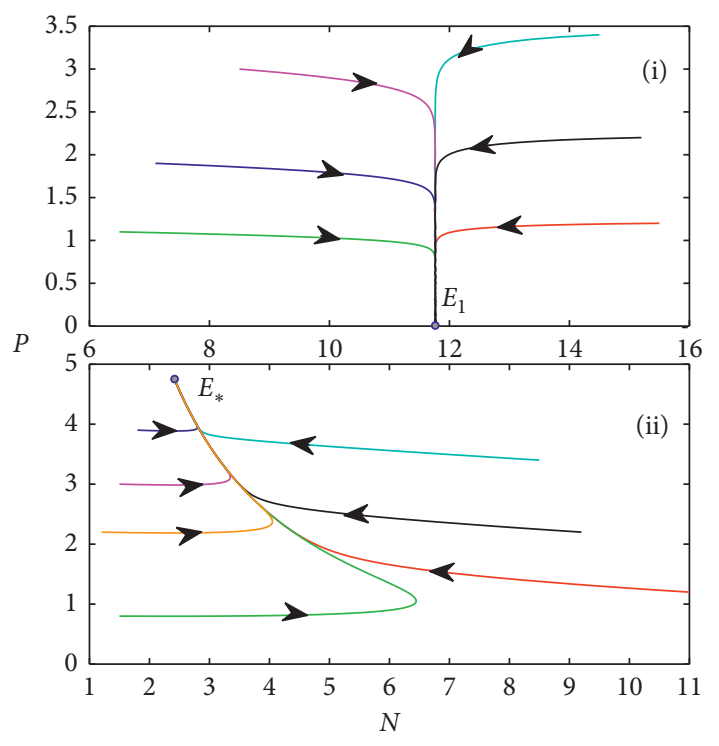

(b)

Figure 3: (a) Bifurcation diagram with respect to $x$ and $I$, where the white area (i) indicates that there exists the only boundary equilibrium $E_{1}$ that is globally asymptotically stable, and the pink area (ii) implies that the positive equilibrium $E_{*}$ is globally asymptotically stable but the boundary equilibrium $E_{1}$ is unstable. (b) The global stability of the equilibria in model (1) with different cell size $x$, in which (i) for the boundary equilibrium $E_{1}$ with $x=0.05$ and (ii) for the positive equilibrium $E_{*}$ with $x=0.51$.

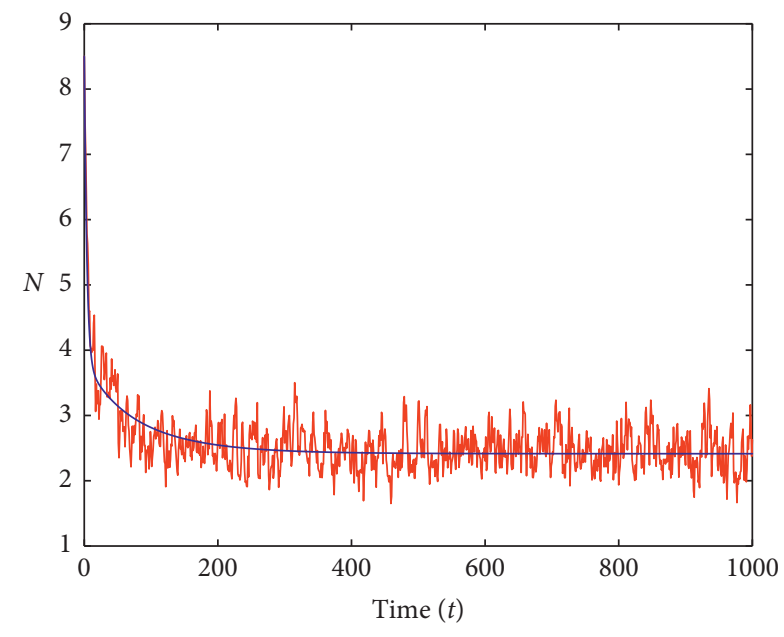

- Stochastic

— Deterministic

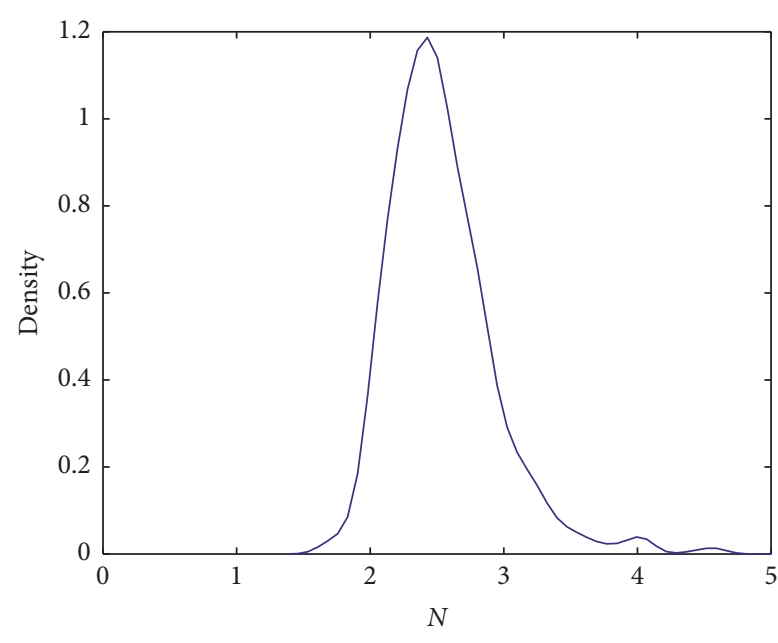

(b)

FIgUre 4: Continued. 


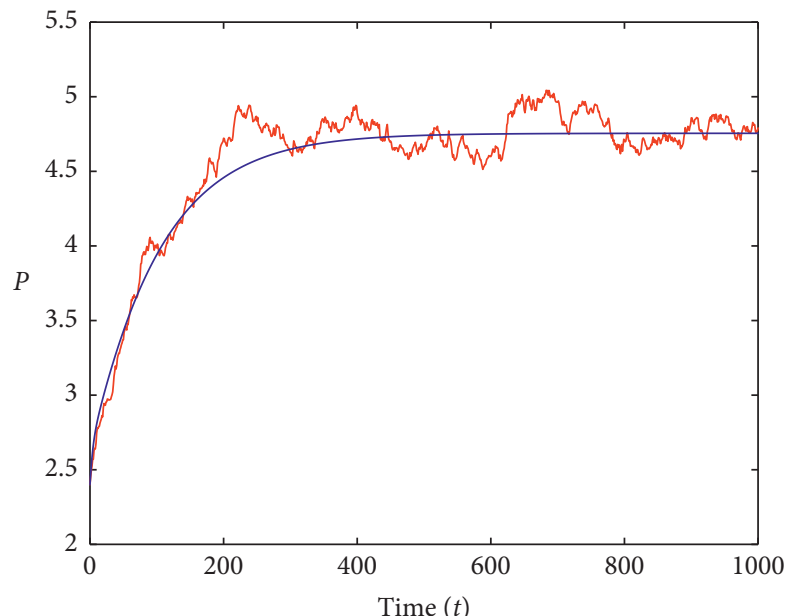

- Stochastic

Deterministic

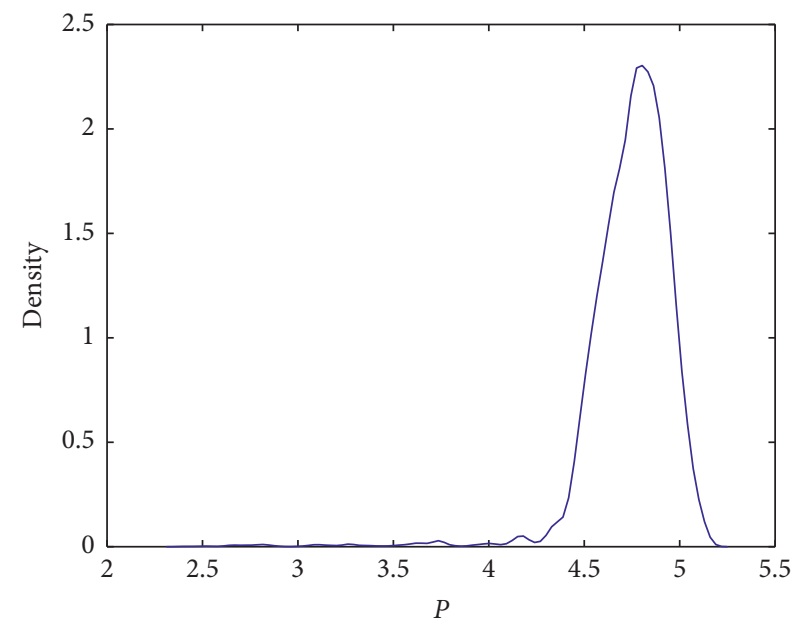

(d)

FIGURE 4: (a) and (c) are the solutions of nutrient and phytoplankton in the stochastic model (3) and its corresponding deterministic model (1) with initial value $\left(N_{0}, P_{0}\right)=(8.5,2.4)$, respectively. (b) and (d) are the probability density function of nutrient and phytoplankton in model (3), respectively.

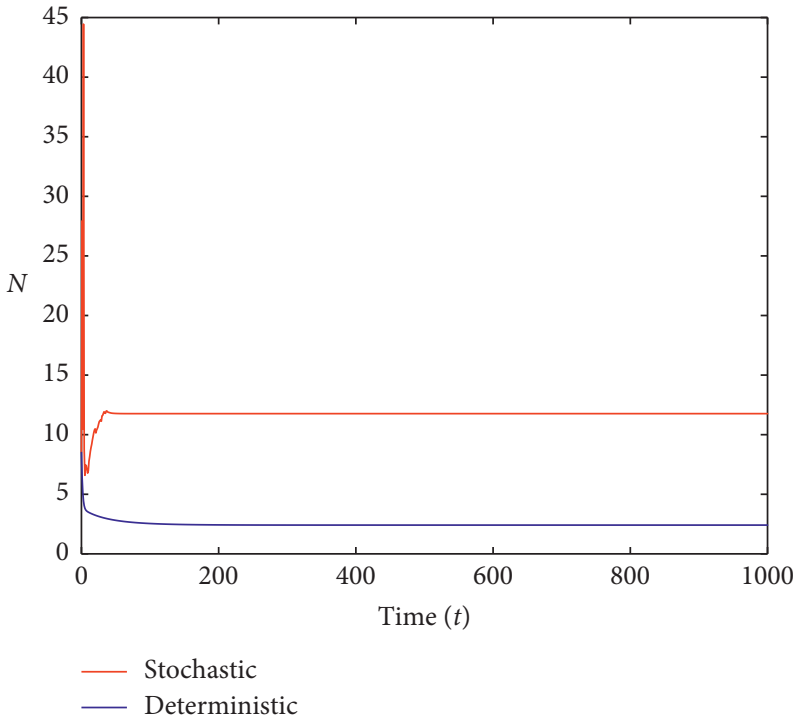

(a)

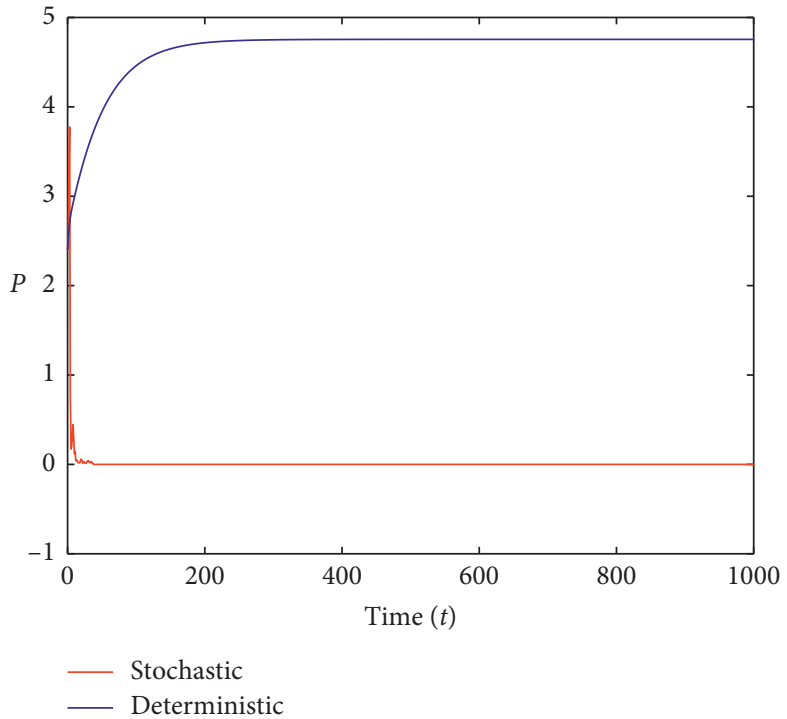

(b)

Figure 5: The solutions of the stochastic model (3) and its corresponding deterministic model (1) with initial value $\left(N_{0}, P_{0}\right)=(8.5,2.4)$. (a) For the nutrient. (b) For the phytoplankton.

which satisfy the parameter condition in Theorem 8 . Hence, there exists a stationary distribution of model (3), as is shown in Figure 7.

In Figure 7(a), one can find that, with the increase of cell size, the height of probability density function of phytoplankton decreases, but that of the probability density function of nutrition increases (see Figure 7(c)), which suggests that the increase of cell size is not conducive to stabilize the nutrient-phytoplankton dynamics in a stochastic sense.

In Figure 7(b), it is shown that the maximum value of the boxplot for phytoplankton increases, while the maximum value of the boxplot for nutrient remains unchanged (see Figure 7(d)), as the cell size increases. This implies that the probability density function of phytoplankton is positively skewed (from left to right), but the probability 


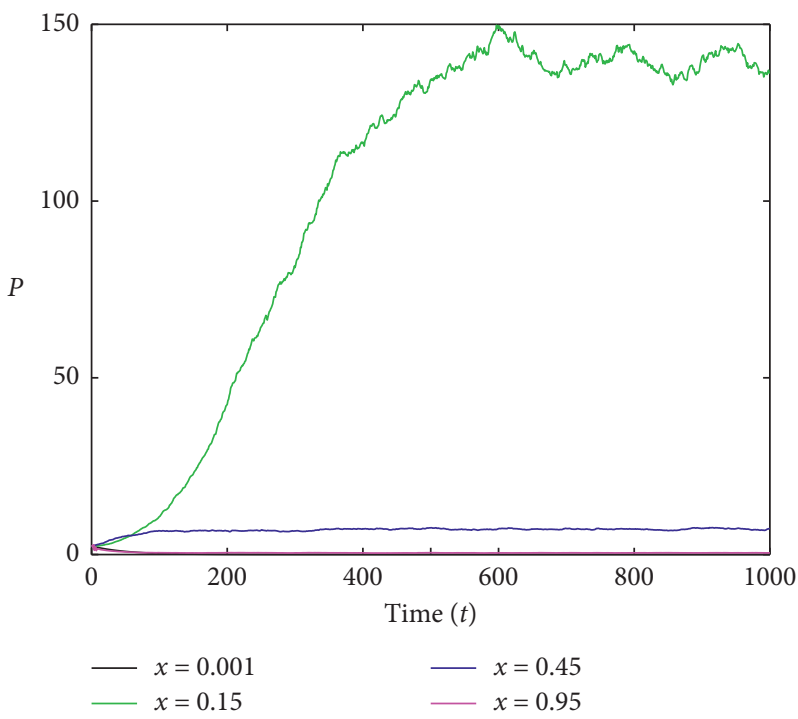

FIGURE 6: The solution of phytoplankton in model (3) with different cell size $x$, where the noise intensity is fixed to $\delta=0.1$, and the black curve is for $x=0.001$, the green curve is for $x=0.15$, the blue curve is for $x=0.45$, and the pink curve is for $x=0.95$.

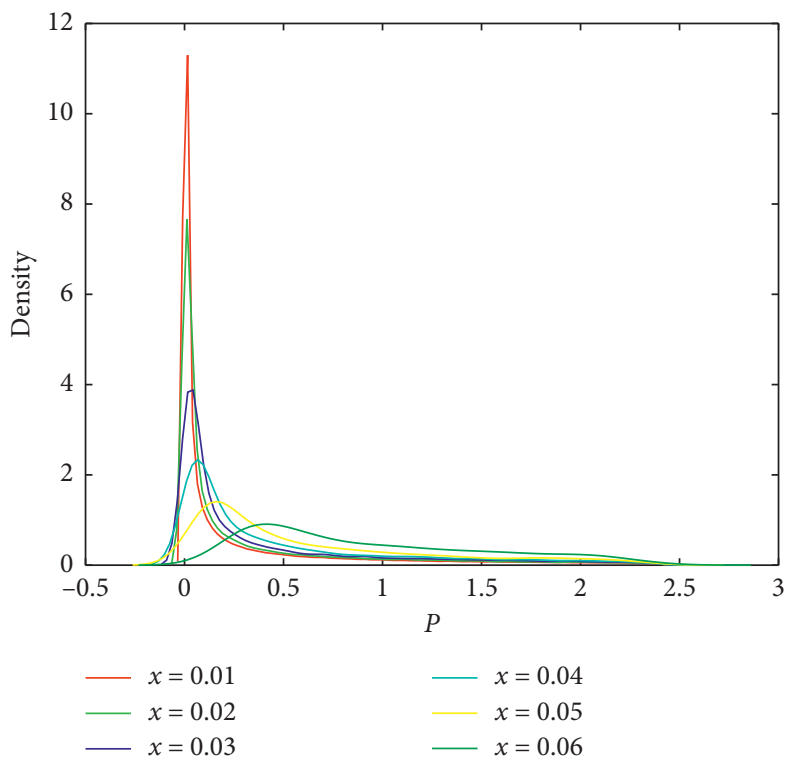

(a)

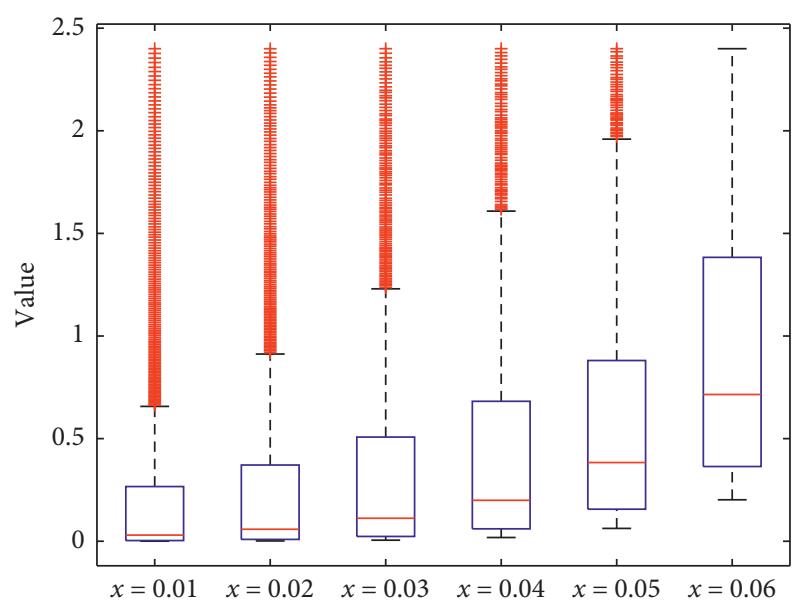

(b)

Figure 7: Continued. 


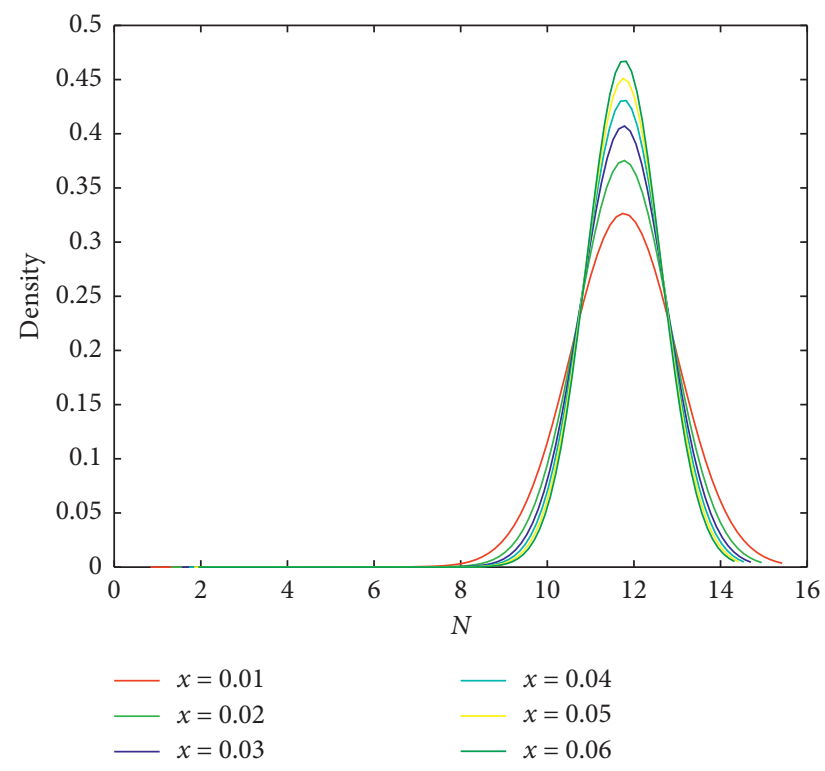

(c)

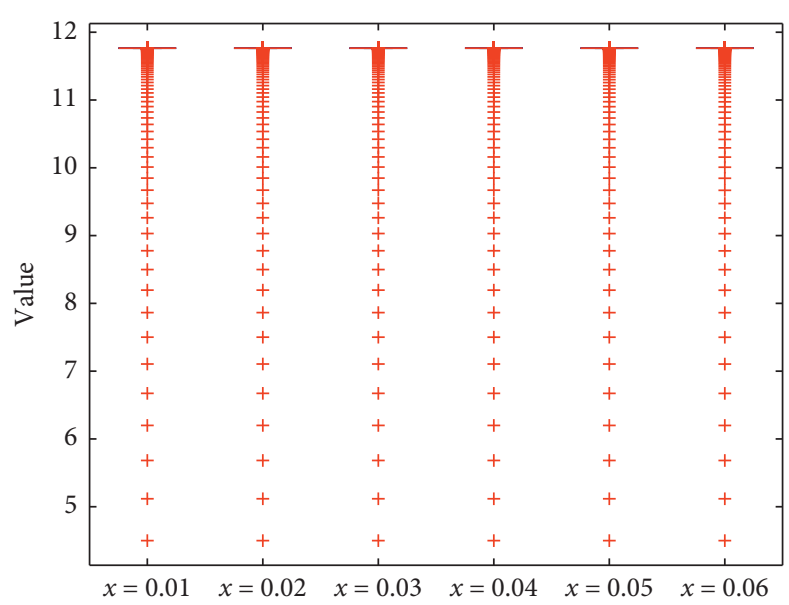

(d)

Figure 7: The effect of cell size on the existence of stationary distribution for model (3) with different cell size $x=0.01,0.02$, $0.03,0.04,0.05,0.06$. (a) and (c) are the probability density function of phytoplankton and nutrient in model (3), respectively. (b) and (d) are the boxplot of the solution for phytoplankton and nutrient in model (3) with the corresponding cell size, respectively.

density function of nutrition is not shifted as the cell size increases.

\section{Conclusion}

In recent years, many field and laboratory evidences indicated that the plankton body size, especially the cell size of phytoplankton, plays a key role in the metabolism, growth, and interaction of phytoplankton [6, 51, 54]. However, little theoretical work has been done in this field. Based on these facts, in this paper, we study analytically and numerically a deterministic nutrient-phytoplankton model taking into account the effects of cell size and its corresponding stochastic version. The consideration of the cell size effect into our proposed model, in this study, makes it different from the traditional nutrient-phytoplankton models $[12,14,15,22,39-45,61]$. The main purpose of this paper is to study the cell size effects on the nutrientphytoplankton dynamics within the deterministic and stochastic environments.

Mathematically, we show that the existence and stability of the equilibria for the deterministic model (1) can be determined by the value of $X$ (i.e., the cell size). In other words, if $X<(\mathrm{ma} / \mathrm{I})$, there exists the only boundary equilibrium that is locally and globally asymptotically stable (see Theorems 2 and 3); if $X<(m a / I)$, the positive equilibrium appears, which is locally and globally asymptotically stable, whereas the boundary equilibrium becomes unstable (see Theorems 2 and 4). For the stochastic model (3), we first prove the existence and uniqueness of the positive solution, and then, the stochastic extinction and persistence in the mean, as well as the existence of ergodic stationary distribution for model (3) are further explored. Ecologically, via numerical simulations, we find that cell size has rich and complex impacts on the nutrient-phytoplankton dynamics in model (1) and model (3) as follow:

(i) If the cell size is smaller or larger $(0<x<$ 0.07807216867 or $x>2.981219088$ ), the phytoplankton cannot survive (see Figures 1(d) and 1(f)). In these cases, the increase of cell size has no impact on the nutrient concentration (see Figures 1(a) and 1(c)). As the cell size increases to the intermediate size, that is, $0.07807216867<x<2.981219088$, the phytoplankton appears and its density as well as nutrient concentration begin to change significantly with cell size.

$\left(i_{1}\right)$ When $0.07807216867<x \leq 0.1$, the increase of cell size can lead to a rapid increase in phytoplankton density and result in a significant reduction in nutrient concentration (see Figures 2(a) and 2(d), respectively). However, the nutrient concentration is still high at this time and the phytoplankton density reached its peak at $x=0.1$ (see Figures $1(\mathrm{e})$ and 2(d)), which denotes that the intermediate cell size is the optimum size for the growth of phytoplankton. This result is consistent with the experimental results obtained in $[6,54]$.

$\left(i_{2}\right)$ When $0.1<x \leq 0.6$, the increase of cell size is capable to decrease the density of phytoplankton and the concentration of nutrient at the same time (see Figures 2(b) and 2(e)). This suggests that this range is most advantageous for reducing the risk of phytoplankton blooms and the possibility of eutrophication of water bodies. Consequently, if the eutrophication of lakes, rivers, and other water bodies, leading to the occurrence of algal blooms, we can invest a number of 
phytoplankton with cell size $x \in(0.1,0.6]$ to possibly control these phenomena.

$\left(i_{3}\right)$ When $0.6<x<2.981219088$, the increase of cell size can cause the significant decrease of phytoplankton and the rapid increase of nutrient concentration (see Figures 2(c) and 2(f), respectively). In this case, the density of phytoplankton is very close to zero as the cell size increases. Actually, based on Theorem 2, the phytoplankton will be extinct and the nutrient concentration will eventually increase to a invariant constant as the cell size continuously increases to greater than 2.981219088. Consequently, the increasing cell size of phytoplankton in this region can inhibit the proliferation of phytoplankton (see Figure 2(f)).

(ii) When the cell size is smaller or larger, i.e., $0<x<0.07807216867$ or $x>2.981219088$, there exists the only boundary equilibrium $E_{1}$ in model (1), which is globally asymptotically stable (see (i) of Figure $3(\mathrm{~b}$ ) and Theorem 3), but the positive equilibrium does not exist in these cases (see Theorem 2); when $0.07807216867<x<2.981219088$, which lies in the intermediate cell size, the positive equilibrium exists and is globally asymptotically stable, while the boundary becomes unstable (see (ii) of Figure 3(b) and Theorem 4). Hence, if we can properly control the cell size of phytoplankton to make phytoplankton and nutrient coexist stably, the phytoplankton will not grow rapidly in large quantities; thus, phytoplankton blooms may not occur, which is consistent with the conclusion in $\left(i_{2}\right)$ and $\left(i_{3}\right)$.

(iii) With a fixed value of cell size $x$, the smaller stochastic environmental fluctuations are shown to be unable to affect the persistence of model (3) (see Figure 4 and Theorem 6), but the larger stochastic environmental fluctuations can result in the extinction of phytoplankton with probability one (see Figure 5 and Theorem 7). With a fixed value of noise intensity, the smaller or larger cell size is found to be capable of causing the extinction of phytoplankton, while the intermediate cell size is in favor of the persistence of phytoplankton (see Figure 6). By comparison, the smaller random environmental disturbances and the intermediate cell size have similar positive effects on the persistence of phytoplankton, but the larger cell size, smaller cell size, and larger random environmental disturbances have similar positive effects on the extinction of phytoplankton.

(iv) For the existence of the stationary distribution for model (3), as the cell size increases, the probability density function of phytoplankton has a positive shift and its height is decreasing, but the probability density function of nutrition has hardly moved and its height is increasing (see Figure 7). This indicates that the cell size may be capable to significantly affect the distribution of phytoplankton in water bodies.

In view of the above findings, the questions raised in Section 1 have been partially well answered. Furthermore, in comparison to the results with respect to cell size obtained in [55-57], the results of this paper are richer and more complex. Though our study cannot directly confirm that cell size is a factor inducing the phytoplankton blooms, we believe that cell size has the potential to influence the formation of this phenomena because it can affect the growth of phytoplankton and the variation of nutrient concentration in the aquatic ecosystems. Hence, the motive of this paper is not to give a feasible theoretical explanation for the sizedependent mechanisms of phytoplankton growth but to provide a new insight into understanding the intrinsic law of the nutrient-phytoplankton dynamics.

\section{Data Availability}

The data used to support the findings of this study are included within the article.

\section{Conflicts of Interest}

The authors declare that they have no conflicts of interest.

\section{Acknowledgments}

This work was supported by the National Key Research and Development Program of China (grant no. 2018YFE0103700) and the National Natural Science Foundation of China (grant nos. 61871293 and 31570364).

\section{References}

[1] J. Huisman, G. A. Codd, H. W. Paerl, B. W. Ibelings, J. M. H. Verspagen, and P. M. Visser, "Cyanobacterial blooms," Nature Reviews Microbiology, vol. 16, no. 8, pp. 471-483, 2018.

[2] C. J. Gobler, O. M. Doherty, T. K. Hattenrath-Lehmann, A. W. Griffith, Y. Kang, and R. W. Litaker, "Ocean warming since 1982 has expanded the niche of toxic algal blooms in the North Atlantic and North Pacific oceans," Proceedings of the National Academy of Sciences, vol. 114, no. 19, pp. 4975-4980, 2017.

[3] L. Guo, "Ecology: doing battle with the green monster of Taihu lake," Science, vol. 317, no. 5842, p. 1166, 2007.

[4] A. M. Michalak, E. J. Anderson, D. Beletsky et al., "Recordsetting algal bloom in lake Erie caused by agricultural and meteorological trends consistent with expected future conditions," Proceedings of the National Academy of Sciences, vol. 110, no. 16, pp. 6448-6452, 2013.

[5] A. Burson, M. Stomp, E. Greenwell, J. Grosse, and J. Huisman, "Competition for nutrients and light: testing advances in resource competition with a natural phytoplankton community," Ecology, vol. 99, no. 5, pp. 1108-1118, 2018.

[6] E. Marañón, P. Cermeño, D. C. López-Sandoval et al., "Unimodal size scaling of phytoplankton growth and the size dependence of nutrient uptake and use," Ecology Letters, vol. 16, no. 3, pp. 371-379, 2013.

[7] H. Ullah, I. Nagelkerken, S. U. Goldenberg, and D. A. Fordham, "Climate change could drive marine food web collapse through altered trophic flows and cyanobacterial proliferation," PLoS Biology, vol. 16, no. 1, Article ID e2003446, 2018. 
[8] X. Jiang, H. Gao, L. Zhang, H. Liang, and X. Zhu, "Rapid evolution of tolerance to toxic microcystis in two cladoceran grazers," Sci. Rep.vol. 6, p. 25319, 2016.

[9] G. Sandrini, X. Ji, J. M. H. Verspagen et al., "Rapid adaptation of harmful cyanobacteria to rising $\mathrm{CO}_{2}$," Proceedings of the National Academy of Sciences, vol. 113, no. 33, pp. 9315-9320, 2016.

[10] D. J. Conley, H. W. Paerl, R. W. Howarth et al., "Ecology: controlling eutrophication: nitrogen and phosphorus," Science, vol. 323, no. 5917, pp. 1014-1015, 2009.

[11] J. Rapala and K. Sivonen, "Assessment of environmental conditions that favor hepatotoxic and neurotoxic anabaena spp. strains cultured under light limitation and different temperatures," Microbial Ecology, vol. 36, no. 2, pp. 181-192, 1998.

[12] A. Huppert, B. Blasius, and L. Stone, "A model of phytoplankton blooms," The American Naturalist, vol. 159, no. 2, pp. 156-171, 2002.

[13] J. Chattopadhyay, R. R. Sarkar, and S. Mandal, “Toxin producing plankton may act as a biological control for planktonic blooms-field study and mathematical modelling," Journal of Theoretical Biology, vol. 215, no. 3, pp. 333-344, 2002.

[14] Z. Qiu and H. Zhu, "Complex dynamics of a nutrientplankton system with nonlinear phytoplankton morality and allelopathy," Discrete and Continuous Dynamical Systems-Series B, vol. 21, no. 8, pp. 2703-2728, 2016.

[15] C. J. Dai, M. Zhao, H. G. Yu, and Y. P. Wang, "Delay-induced instability in a nutrient-phytoplankton system with flow," Physical Review E, vol. 91, no. 3, Article ID 032929, 2015.

[16] T. Saha and M. Bandyopadhyay, "Dynamical analysis of toxin producing phytoplankton-zooplankton interactions," Nonlinear Analysis: Real World Applications, vol. 10, no. 1, pp. 314-332, 2009.

[17] R. Pal, D. Basu, and M. Banerjee, "Modelling of phytoplankton allelopathy with monod-haldane-type functional response-a mathematical study," Biosystems, vol. 95, no. 3, pp. 243-253, 2009.

[18] H. Yu, M. Zhao, and R. P. Agarwal, "Stability and dynamics analysis of time delayed eutrophication ecological model based upon the zeya reservoir," Mathematics and Computers in Simulation, vol. 97, pp. 53-67, 2014.

[19] B. Ghanbari and J. F. Gómez-Aguilar, "Modeling the dynamics of nutrient-phytoplankton-zooplankton system with variable-order fractional derivatives," Chaos, Solitons \& Fractals, vol. 116, pp. 114-120, 2018.

[20] J.-F. Zhang, S. Wang, and X. Kong, "Effects of toxin delay on the dynamics of a phytoplankton-zooplankton model," Physica A: Statistical Mechanics and Its Applications, vol. 505, pp. 1150-1162, 2018.

[21] R. Han and B. Dai, "Spatiotemporal pattern formation and selection induced by nonlinear cross-diffusion in a toxicphytoplankton-zooplankton model with Allee effect," Nonlinear Analysis: Real World Applications, vol. 45, pp. 822-853, 2019.

[22] C. J. Dai, H. G. Yu, Q. Guo et al., "Dynamics induced by delay in a nutrient-phytoplankton model with multiple delays," Complexity, vol. 2019, Article ID 3879626, 16 pages, 2019.

[23] H. Yu, M. Zhao, Q. Wang, and R. P. Agarwal, "A focus on long-run sustainability of an impulsive switched eutrophication controlling system based upon the zeya reservoir," Journal of the Franklin Institute, vol. 351, no. 1, pp. 487-499, 2014.

[24] J. Yang and M. Zhao, "A mathematical model for the dynamics of a fish algae consumption model with impulsive control strategy," Journal of Applied Mathematics, vol. 2012, Article ID 452789, 17 pages, 2012.

[25] A. Huppert, B. Blasius, R. Olinky, and L. Stone, "A model for seasonal phytoplankton blooms," Journal of Theoretical Biology, vol. 236, no. 3, pp. 276-290, 2005.

[26] Y. Cai, Z. Gui, X. Zhang, H. Shi, and W. Wang, "Bifurcations and pattern formation in a predator-prey model," International Journal of Bifurcation and Chaos, vol. 28, no. 11, p. 1850140, 2018.

[27] H. Zhang, Y. Cai, S. Fu, and W. Wang, "Impact of the fear effect in a prey-predator model incorporating a prey refuge," Applied Mathematics and Computation, vol. 356, pp. 328-337, 2019.

[28] J. Wang, Y. L. Cai, S. M. Fu, and W. M. Wang, "The effect of the fear factor on the dynamics of a predator-prey model incorporating the prey refuge," Chaos: An Interdisciplinary Journal of Nonlinear Science, vol. 29, no. 8, Article ID 083109, 2019.

[29] B. Yang, Y. Cai, K. Wang, and W. Wang, "Optimal harvesting policy of logistic population model in a randomly fluctuating environment," Physica A: Statistical Mechanics and Its Applications, vol. 526, Article ID 120817, , 2019.

[30] W. Wang, X. Gao, Y. Cai, H. Shi, and S. Fu, "Turing patterns in a diffusive epidemic model with saturated infection force," Journal of the Franklin Institute, vol. 355, no. 15, pp. 72267245, 2018.

[31] H. Liu, H. Yu, C. Dai et al., "Dynamic analysis of a reactiondiffusion impulsive hybrid system," Nonlinear Analysis: Hybrid Systems, vol. 33, pp. 353-370, 2019.

[32] Y. Cai, Y. Kang, M. Banerjee, and W. Wang, "A stochastic sirs epidemic model with infectious force under intervention strategies," Journal of Differential Equations, vol. 259, no. 12, pp. 7463-7502, 2015.

[33] Y. Cai, Y. Kang, and W. Wang, "A stochastic sirs epidemic model with nonlinear incidence rate," Applied Mathematics and Computation, vol. 305, pp. 221-240, 2017.

[34] Y. Cai, J. Jiao, Z. Gui, Y. Liu, and W. Wang, "Environmental variability in a stochastic epidemic model," Applied Mathematics and Computation, vol. 329, pp. 210-226, 2018.

[35] M. Zhao, X. Wang, H. Yu, and J. Zhu, "Dynamics of an ecological model with impulsive control strategy and distributed time delay," Mathematics and Computers in Simulation, vol. 82, no. 8, pp. 1432-1444, 2012.

[36] M. Zhao, H. Yu, and J. Zhu, "Effects of a population floor on the persistence of chaos in a mutual interference host-parasitoid model," Chaos, Solitons \& Fractals, vol. 42, no. 2, pp. 1245-1250, 2009.

[37] C. Dai, M. Zhao, and L. Chen, "Complex dynamic behavior of three-species ecological model with impulse perturbations and seasonal disturbances," Mathematics and Computers in Simulation, vol. 84, pp. 83-97, 2012.

[38] G. A. Riley, H. Stommel, and D. P. Burrpus, "Quantitative ecology of the plankton of the western North Atlantic," Bulletin-Bingham Oceanographic Collection, vol. 12, pp. 1169, 1949.

[39] C. Dai, M. Zhao, and H. Yu, "Dynamics induced by delay in a nutrient-phytoplankton model with diffusion," Ecological Complexity, vol. 26, pp. 29-36, 2016.

[40] A. Sharma, A. K. Sharma, and K. Agnihotri, "The dynamic of plankton-nutrient interaction with delay," Applied Mathematics and Computation, vol. 231, pp. 503-515, 2014.

[41] A. Chatterjee, S. Pal, and S. Chatterjee, "Bottom up and top down effect on toxin producing phytoplankton and its consequence on the formation of plankton bloom," Applied 
Mathematics and Computation, vol. 218, no. 7, pp. 3387-3398, 2011.

[42] S. Ruan, "Oscillations in plankton models with nutrient recycling," Journal of Theoretical Biology, vol. 208, no. 1, pp. 15-26, 2001.

[43] S. Chen, X. Chen, Y. Peng, and K. Peng, "A mathematical model of the effect of nitrogen and phosphorus on the growth of blue-green algae population," Applied Mathematical Modelling, vol. 33, no. 2, pp. 1097-1106, 2009.

[44] S. Pal, S. Chatterjee, and J. Chattopadhyay, "Role of toxin and nutrient for the occurrence and termination of plankton bloom-results drawn from field observations and a mathematical model," Biosystems, vol. 90, no. 1, pp. 87-100, 2007.

[45] S. Chakraborty, P. K. Tiwari, A. K. Misra, and J. Chattopadhyay, "Spatial dynamics of a nutrient-phytoplankton system with toxic effect on phytoplankton," Mathematical Biosciences, vol. 264, pp. 94-100, 2015.

[46] Z. Jiang, X. H. Bi, T. Zhang, and B. G. S. A. Pradeep, "Global Hopf bifurcation of a delayed phytoplankton-zooplankton system considering toxin producing effect and delay dependent coefficient," Mathematical Biosciences and Engineering, vol. 16, no. 5, pp. 3807-3829, 2019.

[47] Z. Jiang, W. Zhang, J. Zhang, and T. Zhang, "Dynamical analysis of a phytoplankton-zooplankton system with harvesting term and holling III functional response," International Journal of Bifurcation and Chaos, vol. 28, no. 13, p. 1850162, 2018.

[48] A. Verdy, F. M., and M. Follows, "Optimal phytoplankton cell size in an allometric model," Marine Ecology Progress Series, vol. 379, pp. 1-12, 2009

[49] Z. V. Finkel, J. Beardall, K. J. Flynn, A. Quigg, T. A. V. Rees, and J. A. Raven, "Phytoplankton in a changing world: cell size and elemental stoichiometry," Journal of Plankton Research, vol. 32, no. 1, pp. 119-137, 2010.

[50] E. P. Y. Tang, "The allometry of algal growth rates," Journal of Plankton Research, vol. 17, no. 6, pp. 1325-1335, 1995.

[51] J. E. Cohen, T. Jonsson, and S. R. Carpenter, "Ecological community description using the food web, species abundance, and body size," Proceedings of the National Academy of Sciences (USA), vol. 100, no. 4, pp. 17810-1786, 2003.

[52] K. G. Porter, "Selective grazing and differential digestion of algae by zooplankton," Nature, vol. 244, no. 5412, pp. 179-180, 1973.

[53] D. Blasco, T. T. Packard, and P. G. Coble, "Size dependence of growth rate, respiratory electron transport system activity, and chemical composition in marine diatoms in the laboratory," Journal of Phycology, vol. 18, no. 1, pp. 58-63, 2004.

[54] B. Bec, Y. Collos, A. Vaquer, D. Mouillot, and P. Souchu, "Growth rate peaks at intermediate cell size in marine photosynthetic picoeukaryotes," Limnology and Oceanography, vol. 53, no. 2, pp. 863-867, 2008.

[55] L. Jiang, O. M. E. Schofield, and P. G. Falkowski, "Adaptive evolution of phytoplankton cell size," The American Naturalist, vol. 166, no. 4, pp. 496-505, 2005.

[56] Z. Pu, M. H. Cortez, and L. Jiang, "Predator-prey coevolution drives productivity-richness relationships in planktonic systems," The American Naturalist, vol. 189, no. 1, pp. 28-42, 2017.

[57] Q. Zhao, S. Liu, and D. Tian, "Dynamic behavior analysis of phytoplankton-zooplankton system with cell size and time delay," Chaos, Solitons \& Fractals, vol. 113, pp. 160-168, 2018.

[58] H. Serizawa, T. Amemiya, and K. Itoh, "Patchiness in a minimal nutrient-phytoplankton model," Journal of Biosciences, vol. 33, no. 3, pp. 391-403, 2008.
[59] T. C. Gard, "Stability for multispecies population models in random environments," Nonlinear Analysis: Theory, Methods \& Applications, vol. 10, no. 12, pp. 1411-1419, 1986.

[60] R. May, Stability and Complexity in Model Ecosystems, Princeton University Press, New York, USA, 2001.

[61] X. Yu, S. Yuan, and T. Zhang, "The effects of toxin-producing phytoplankton and environmental fluctuations on the planktonic blooms," Nonlinear Dynamics, vol. 91, no. 3, pp. 1653-1668, 2018.

[62] Z. Huang and G. Huang, "Mathematical analysis on deterministic and stochastic lake ecosystem models," Mathematical Biosciences and Engineering, vol. 16, no. 5, pp. 4723-4740, 2019.

[63] B. I. Camara, R. Yamapi, and H. Mokrani, "Environmental stochastic effects on phytoplankton-zooplankton dynamics," Nonlinear Dynamics, vol. 96, no. 3, pp. 2013-2029, 2019.

[64] D. Valenti, G. Denaro, B. Spagnolo et al., "Stochastic models for phytoplankton dynamics in Mediterranean sea," Ecological Complexity, vol. 27, pp. 84-103, 2016.

[65] C. Xu and S. Yuan, "An analogue of break-even concentration in a simple stochastic chemostat model," Applied Mathematics Letters, vol. 48, pp. 62-68, 2015.

[66] G. Brikhoff and G. Rota, Ordinary Differential Equations, John Wiley \& Sons, New York, USA, 1982.

[67] X. Mao, Stochastic Differential Equations and Applications, Horwood Publishing, Chichester, UK, 2nd edition, 2007.

[68] T. Gard, Introduction to Stochastic Differential Equations, Marcel Dekker, New York, USA, 1988.

[69] X. Mao, Stochastic Differential Equations and Their Applications, Horwood, Chichester, UK, 1997.

[70] M. Liu, K. Wang, and Q. Wu, "Survival analysis of stochastic competitive models in a polluted environment and stochastic competitive exclusion principle," Bulletin of Mathematical Biology, vol. 73, no. 9, pp. 1969-2012, 2011.

[71] R. Z. Hasminiskii, Stochastic Stability of Differential Equations, Sijthoff and Noordhoff, Alphen aan den Rijn, Netherlands, 1980.

[72] G. T. Evans and J. S. Parslow, "A model of annual plankton cycles,” Biological Oceanography, vol. 3, pp. 327-347, 1985.

[73] D. J. Higham., "An algorithmic introduction to numerical simulation of stochastic differential equations," SIAM Review, vol. 43, no. 3, pp. 525-546, 2001. 


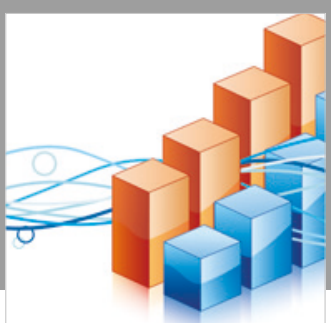

Advances in

Operations Research

\section{-n-m}
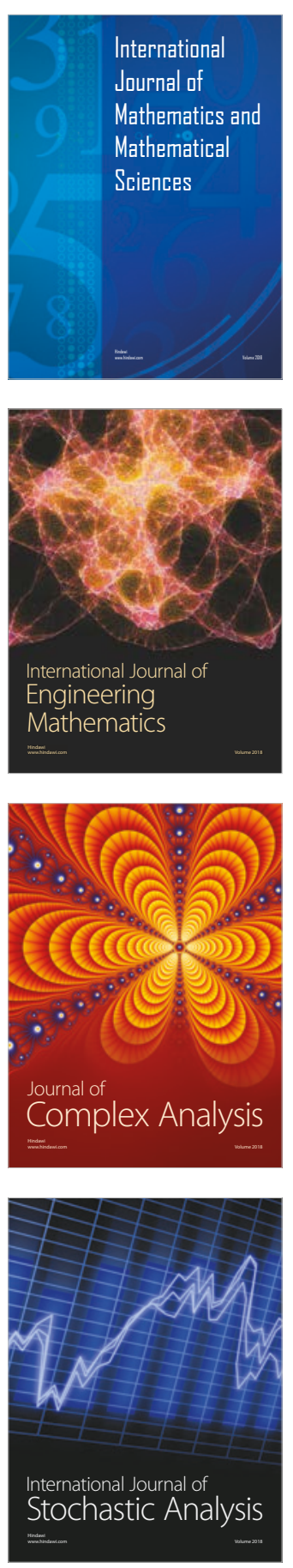
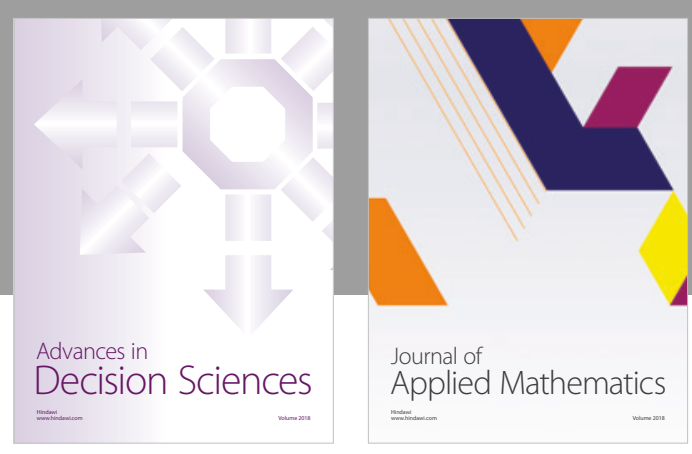

Journal of

Applied Mathematics
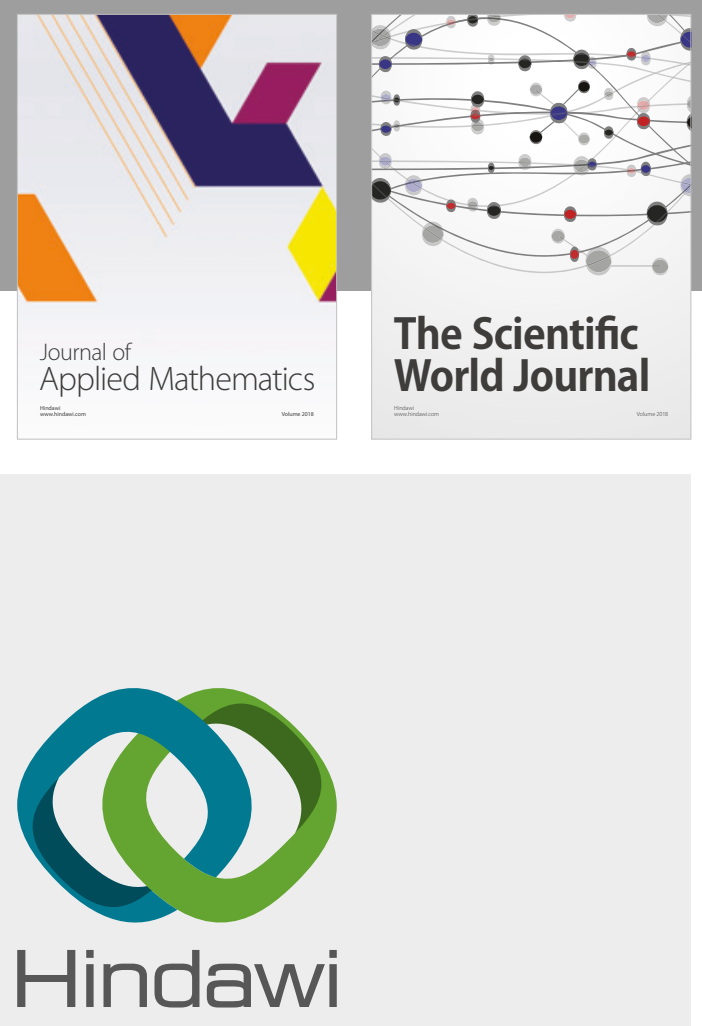

Submit your manuscripts at

www.hindawi.com

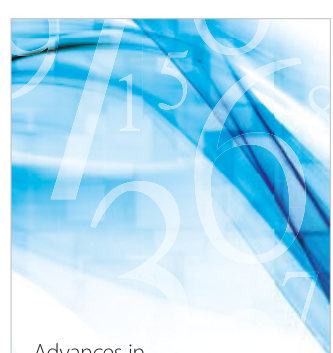

Advances in
Numerical Analysis
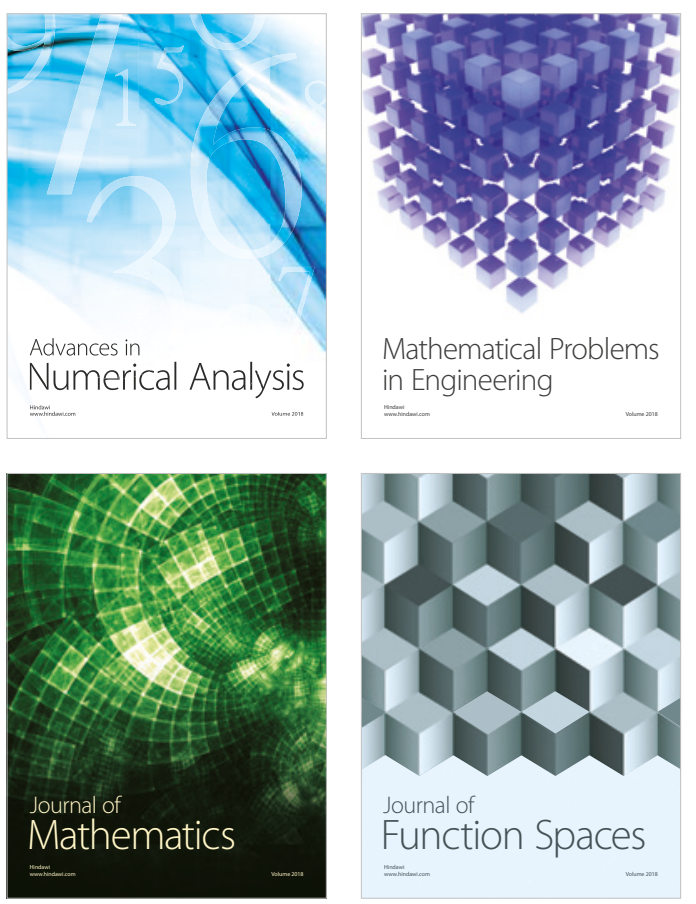

Mathematical Problems in Engineering

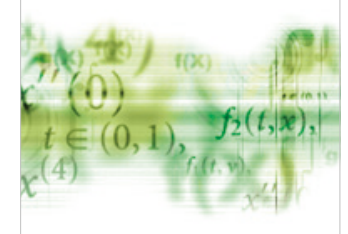

International Journal of

Differential Equations

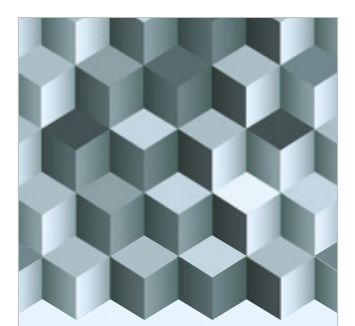

Journal of

Function Spaces
The Scientific

World Journal

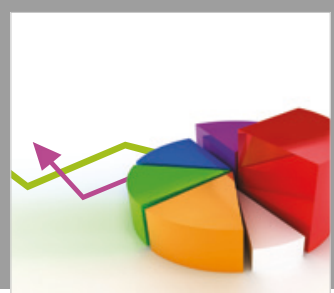

Journal of

Probability and Statistics
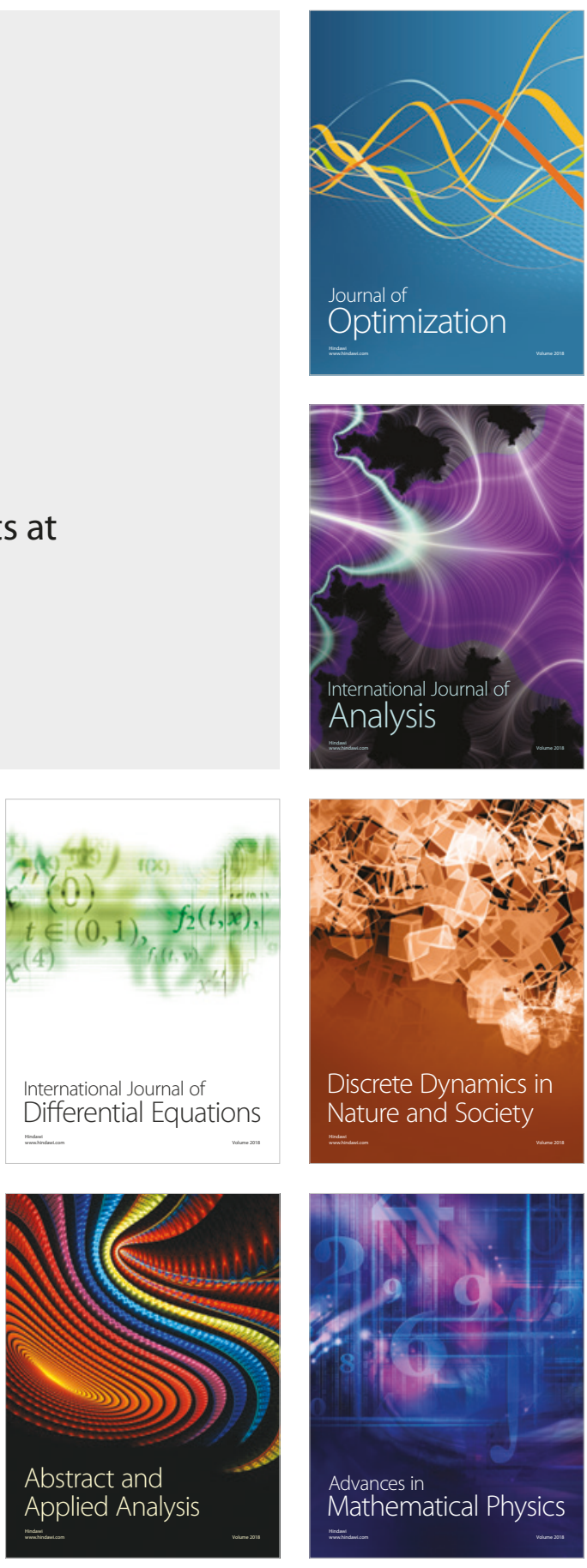